\title{
Raman and $x$-ray absorption spectroscopy study of the phase evolution induced by mechanical milling and thermal treatments in $R_{2} \mathrm{Ti}_{2} \mathrm{O}_{7}$ pyrochlores
}

\author{
M. L. Sanjuán, ${ }^{1}$ C. Guglieri, ${ }^{1}$ S. Díaz-Moreno, ${ }^{2}$ G. Aquilanti, ${ }^{3}$ A. F. Fuentes,${ }^{4}$ L. Olivi, ${ }^{3}$ and J. Chaboy ${ }^{1, *}$ \\ ${ }^{1}$ Instituto de Ciencia de Materiales de Aragón and Departamento de Física de la Materia Condensada, \\ Consejo Superior de Investigaciones Científicas, Universidad de Zaragoza, 50009 Zaragoza, Spain \\ ${ }^{2}$ Diamond Light Source Ltd, Harwell Science and Innovation Campus, Chilton, Didcot, Oxfordshire, OX11 ODE, UK \\ ${ }^{3}$ Sincrotrone Trieste, ELETTRA, 34149 Basovizza (TS), Italy \\ ${ }^{4}$ CINVESTAV-Unidad Saltillo, Apartado Postal 663, 25000-Saltillo, Coahuila, Mexico
}

(Received 15 February 2011; published 13 September 2011)

\begin{abstract}
We report here a study of the local order in $\mathrm{R}_{2} \mathrm{Ti}_{2} \mathrm{O}_{7},(\mathrm{R}=\mathrm{Y}, \mathrm{Gd}$, and Dy) pyrochlores obtained by mechanical milling (MM) performed by combining both Raman and x-ray absorption (XAS) spectroscopies. In addition to both as-milled and annealed MM samples, measurements have been also recorded by performing an in situ thermal treatment of the MM pyrochlores in the range from ambient to $1300^{\circ} \mathrm{C}$. The analysis of both Raman and XAS data yields a disentangled view of the evolution of the different degree of structural disorder at the Ti and rare-earth sites of the MM samples with the thermal treatment. Moreover, this study allowed us to follow the structural transformation of the MM samples through the region where exothermic processes have been previously observed. Our experiments show that an average fluorite-like structure is never formed in these pyrochlores. As milled-samples are better described as if formed by nanometer-sized pyrochlore domains embedded in a fluorite-like matrix. Thermal annealing induces domain growth, so that the event observed by differential thermal analysis can be attributed to an evolution from medium to long-range pyrochlore ordering, typically from tens to hundreds of nanometers.
\end{abstract}

DOI: 10.1103/PhysRevB.84.104207

PACS number(s): 78.70.Dm, 78.30.-j, 63.20.-e, 61.05.cj

\section{INTRODUCTION}

Pyrochlores are a very large family of compounds with potential application in many areas of technological interest. Because composition electroneutrality can be achieved by a large combination of cation species, pyrochlore oxides $\mathrm{A}_{2} \mathrm{~B}_{2} \mathrm{O}_{7}$ show a great variety of interesting electrical, magnetic, or thermal properties. ${ }^{1-3}$ Due to their chemical stability and their ability to accept rare-earth elements in solid solutions, they are currently regarded as important ceramic matrices for the immobilization of high-level radioactive waste. ${ }^{4}$ The pyrochlore structure is closely related to the fluorite structure $\mathrm{AX}_{2}$, except that there are two cation sites, one-eighth of the anions are absent, and anions are also distributed between two crystallographically non-equivalent positions instead of only one as in fluorites. However, this vacancy might be partially occupied in defect oxides; i.e., different degrees of disorder are possible in both the cation and anion sublattices by using the appropriate chemical substitutions and/or powder processing methods. Many of the above mentioned properties are very much influenced by ion distribution (structural ordering/disordering) and site occupancies. ${ }^{1}$ Thus, $\mathrm{Gd}_{2} \mathrm{Ti}_{2} \mathrm{O}_{7}$ is an ordered pyrochlore and insulating material at all temperatures, while its $\mathrm{Zr}$ analog, $\mathrm{Gd}_{2} \mathrm{Zr}_{2} \mathrm{O}_{7}$, presents a highly disordered atomic array and is a high-temperature oxygen ion conductor material.

The real mechanism of the ordering/disordering processes taking place in pyrochlore-type rare-earth oxides are still the subject of some controversy with different authors arriving at contradictory conclusions. This problem also extends to the case of $\mathrm{R}_{2} \mathrm{Ti}_{2} \mathrm{O}_{7}$ pyrochlores obtained by mechanical milling $(\mathrm{MM})$ and showing different degree of structural disorder. Initially conceived as a method to prepare nanocrystalline metals and powder alloys, MM has also been used for the synthesis of novel crystalline ceramic phases, the preparation of a fine dispersion of second-phase particles or extended solid solutions. ${ }^{5}$ As a far-from-equilibrium processing method, $\mathrm{MM}$ allows the room-temperature preparation of metastable phases existing at equilibrium only at high temperature and/or high pressure and frequently leads to unique defect structures which are difficult to obtain or that are unattainable by any other synthetic route. Upon thermal activation (e.g., postmilling thermal treatments), many of these highly-defective materials exhibit a sequence of transient crystalline phases before reaching the equilibrium configuration. This offers the possibility of exploring the dependence of properties on structure at fixed composition.

A previous work on $\mathrm{R}_{2} \mathrm{Ti}_{2} \mathrm{O}_{7}$ MM pyrochlores showed an $\mathrm{x}$-ray power diffraction (XRD) pattern typical of a poorly crystalline product, although the main reflections of cubic pyrochlores were evident. ${ }^{6}$ With prolonged milling, the number and intensity of the characteristic pyrochlore reflections increased. Independently of the milling time, the differential thermal analysis (DTA) showed the presence of an exothermic event at temperatures close to $800^{\circ} \mathrm{C}$ which was tentatively attributed to the ordering of the anion sublattice. ${ }^{7}$ The existence of such an event could be associated with different phenomena such as crystallization of the pyrochlore from a more or less amorphous matrix or with an order-disorder transition. However, the difficulties in analyzing the oxygen substructure by XRD together with the anomalously high-cross section of $\mathrm{Gd}$ for absorption of thermal as well as cold neutrons, makes it necessary to use another approach for analyzing the $\mathrm{R}$ and $\mathrm{Ti}$ environment in these titanates. The characterization of the degree of structural disorder and the determination of the exact nature of the exothermic event noticed in the DTA curves in these MM pyrochlores needs techniques sensitive to the local structure. 
To address this issue, we have combined Raman and $\mathrm{X}$-ray absorption spectroscopy (XAS), covering both the X-ray absorption near edge structure (XANES) and extended x-ray absorption fine structure (EXAFS) regions, to the study of $\mathrm{Y}_{2} \mathrm{Ti}_{2} \mathrm{O}_{7}, \mathrm{Gd}_{2} \mathrm{Ti}_{2} \mathrm{O}_{7}$, and $\mathrm{Dy}_{2} \mathrm{Ti}_{2} \mathrm{O}_{7} \mathrm{MM}$ pyrochlores, focusing on the occurrence of size effects and on the determination of the local structure around rare-earth and $\mathrm{Ti}$ cations. In addition, the study has been extended to the same compounds obtained by traditional methods to provide a reference of equilibrium site occupancy. To obtain samples with different degrees of ordering/disordering, portions of the as-prepared $\mathrm{MM}$ pyrochlores have been fired at $\mathrm{T}=775^{\circ} \mathrm{C}$ and $\mathrm{T}=$ $900^{\circ} \mathrm{C}$, i.e., below and above the exothermic process observed in the DTA curves. The XAS spectra have been recorded at the Ti $K$-edge in all the compounds, and also at the Gd and Dy $\mathrm{L}_{3}$-edge in $\mathrm{Gd}_{2} \mathrm{Ti}_{2} \mathrm{O}_{7}$ and $\mathrm{Dy}_{2} \mathrm{Ti}_{2} \mathrm{O}_{7}$ samples, respectively, and at the $\mathrm{Y} K$-edge in $\mathrm{Y}_{2} \mathrm{Ti}_{2} \mathrm{O}_{7}$. In addition, Raman spectroscopy and the same XAS measurements have been recorded by performing an in situ thermal treatment in the range from ambient to $1000{ }^{\circ} \mathrm{C}$ on the $\mathrm{MM}$ pyrochlore samples to follow the structural transformation of the sample through the region where the exothermic process appears.

\section{EXPERIMENTAL}

$\mathrm{Y}_{2} \mathrm{Ti}_{2} \mathrm{O}_{7}, \mathrm{Gd}_{2} \mathrm{Ti}_{2} \mathrm{O}_{7}$ and $\mathrm{Dy}_{2} \mathrm{Ti}_{2} \mathrm{O}_{7}$ pyrochlores (hereafter labeled MM) have been obtained at room temperature by mechanical milling. Details of sample preparation and characterization can be found in Ref. 7. The as-milled MM samples have been fired at $\mathrm{T}=775^{\circ} \mathrm{C}$ and $\mathrm{T}=900^{\circ} \mathrm{C}$, hereafter labeled 775-MM and 900-MM samples. In addition, the same pyrochlores have been obtained by traditional solid state methods, hereafter labeled SS. Structural characterization was performed at room temperature by means of powder x-ray diffraction, using a rotating-anode Rigaku diffractometer in the Bragg-Brentano geometry, with $\mathrm{Cu}-\mathrm{K} \alpha$ radiation.

Raman scattering measurements were performed in backscattering geometry, using an optical microprobe spectrometer (Model XY, Dilor, France) with a CCD detector. Spectra were taken at room temperature $(\mathrm{RT})$ with a $\times 50$ microscope objective lens. The power of the laser line was $40 \mathrm{~mW}$ and the spectral resolution $\sim 2 \mathrm{~cm}^{-1}$. Spectra at temperatures between $300 \mathrm{~K}$ and $1173 \mathrm{~K}$ were measured by putting the sample into a LINKAM TS1500 stage with a temperature stability of $0.5 \mathrm{~K}$, and using a $\times 50$ long working distance objective lens. The $514.5 \mathrm{~nm}$ line of an $\mathrm{Ar}^{+}$-ion laser (Model INNOVA 305, Coherent, Palo Alto, CA) was used for sample excitation and the Si Raman line at $520 \mathrm{~cm}^{-1}$ for wavelength calibration.

XAS spectra were recorded at the bending magnet XAFS beamline at the ELETTRA Synchrotron Facility (Trieste, Italy). Experiments were performed at room temperature in transmission mode with ionization chambers (Oxford Instruments) filled with optimal gas mixtures of $\mathrm{He}, \mathrm{N}_{2}, \mathrm{Ar}$, and $\mathrm{Kr}$. The energy of the incoming $\mathrm{x}$-ray beam was tuned with a $\mathrm{Si}(111)$ double-crystal monochromator in the energy range from 4.9 to $19 \mathrm{keV}$ covering the $\mathrm{Ti} K$-edge, $\mathrm{Gd}$, and Dy $\mathrm{L}_{3}$-edges, and the $\mathrm{Y} K$-edge. By using this setup the energy resolution is $\Delta \mathrm{E} / \mathrm{E}=10^{-4}$, i.e., $\sim 0.5 \mathrm{eV}$ at the Ti $K$-edge $(\mathrm{E}=\sim 4.9 \mathrm{keV})$ and $\sim 2 \mathrm{eV}$ at the $\mathrm{Y} K$-edge
$(\mathrm{E}=\sim 17 \mathrm{keV})$. The energy of the Ti and Y $K$-edge XAS spectra was calibrated by measuring the XANES of Ti and $\mathrm{Y}$ metal foils, whereas the $\mathrm{Fe}$ and Co foils were used in the case of the Gd and Dy $\mathrm{L}_{3}$-edges, respectively. Harmonic rejection was achieved by detuning the second crystal from parallel alignment. For the measurements, homogeneous layers of the powdered samples were made by spreading fine powders of the material onto an adhesive tape. Thickness and homogeneity of the samples were optimized to obtain the best signal-to-noise ratio. XANES and EXAFS spectra were also recorded as a function of temperature, from ambient to $1300^{\circ} \mathrm{C}$, using a l'Aquila-Camerino furnace. ${ }^{8}$ For these measurements, samples were prepared as pellets of the pure materials slightly diluted in $\mathrm{BN}$ (boron nitride). The absorption spectra were analyzed according to standard procedures ${ }^{9}$ using the ATHENA program pack. ${ }^{10}$

The ab-initio computation of the XANES spectra was carried out using the multiple-scattering code Continuum ${ }^{11}$ based on the one-electron full-multiple-scattering theory. ${ }^{12}$ A complete discussion of the procedure can be found elsewhere. ${ }^{13,14}$ The potential for the different atomic clusters was approximated by a set of spherically averaged muffin-tin (MT) potentials built by following the standard Mattheis' prescription. The muffin-tin radii were determined following the Norman's criterion. During the computations, care was taken to determine the best choice for the overlap factor between the muffin-tin spheres and for the exchange and correlation components of the final state potential. ${ }^{15}$ The best reproduction of the experimental XANES spectra was obtained by using the Dirac-Hara ECP potential and by imposing a $1 \%$ of overlapping factor. It should be stressed that no free parameter has been used during the calculations. The theoretically calculated spectra have been directly compared to the experimental XANES spectrum i.e., no fitting procedure has been used. The assessment of the quality of the theoretical computations is based on the correct reproduction of the shape and energy position of the different spectral features and of their relative energy separation and the intensity ratio. In all the cases, the theoretical spectra have been convoluted with a Lorentzian shape function $\left(\Gamma_{c}=1.5 \mathrm{eV}\right)$ to account for the core-hole lifetime ${ }^{16}$ and the experimental resolution.

\section{RESULTS AND DISCUSSION}

Considered as a superstructure of the ideal defect fluorite structure with twice the cell constant, the fully ordered or ideal pyrochlore presents cubic symmetry (space group 227) with cations and anions occupying each, two crystallographically different positions. ${ }^{17}$ The larger cation $(\mathrm{R}>1 \AA)$ is normally found at the 8-coordinated A site (16c) whereas the smaller one ( $\mathrm{R} \sim 0.6 \AA$ ) occupies the 6-coordinated B site $(16 d)$. The oxygen atoms fully occupy two different sites, $48 f$ and $8 a$, which are tetrahedrally coordinated by two B and two A cations and by four A cations, respectively. The coordinate of $48 f$ oxygen is $(\mathrm{x}, 1 / 8,1 / 8)$, where $\mathrm{x}$ is an indicator of the degree of disorder in the system. There is another site available for anions in the unit cell, $8 b$, which is tetrahedrally coordinated to four B cations and systematically empty in ideal pyrochlores but might be partially occupied in defect oxides. 

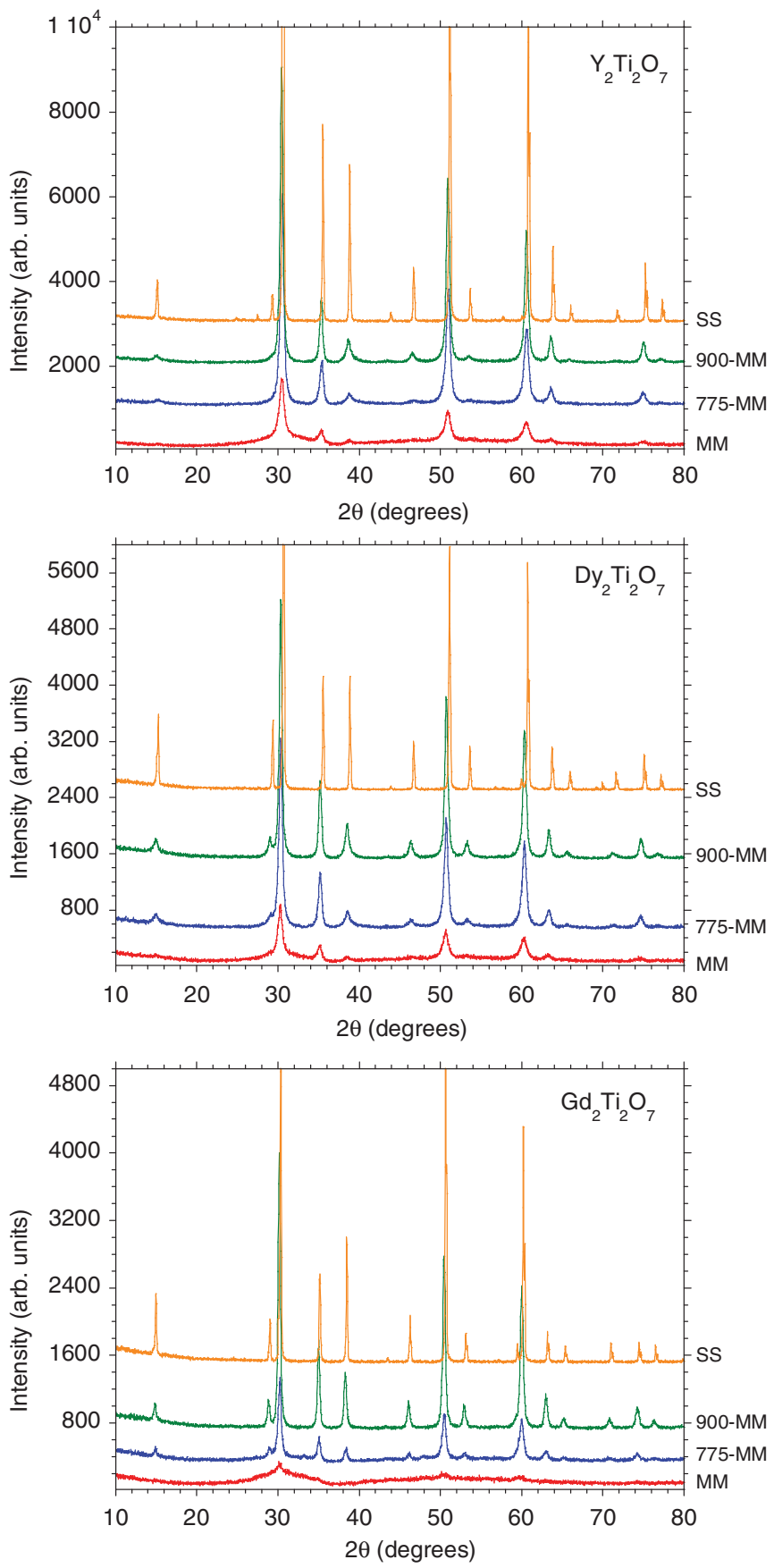

FIG. 1. (Color online) XRD patterns of as-milled (MM) and annealed (775-MM and 900-MM) $\mathrm{Y}_{2} \mathrm{Ti}_{2} \mathrm{O}_{7}, \mathrm{Gd}_{2} \mathrm{Ti}_{2} \mathrm{O}_{7}$, and $\mathrm{Dy}_{2} \mathrm{Ti}_{2} \mathrm{O}_{7}$ samples. For the sake of completeness the XRD patterns of the samples prepared by solid state reaction (SS) are also included.

The $\mathrm{x}$-ray diffractograms of as-milled and annealed samples of $\mathrm{Y}_{2} \mathrm{Ti}_{2} \mathrm{O}_{7}$ (YTO), $\mathrm{Gd}_{2} \mathrm{Ti}_{2} \mathrm{O}_{7}$ (GTO), and $\mathrm{Dy}_{2} \mathrm{Ti}_{2} \mathrm{O}_{7}$ (DTO) are shown in Fig. 1, together with those of SS reference samples. Because of the limited information obtained from XRD on the local structure in disordered systems, especially when light elements as oxygen are involved, a detailed fit of the XRD patterns has not been attempted. We rather limit our discussion to a qualitative description of the diffractograms and the changes occurring upon increasing the annealing temperature. Lattice parameters, selected linewidths
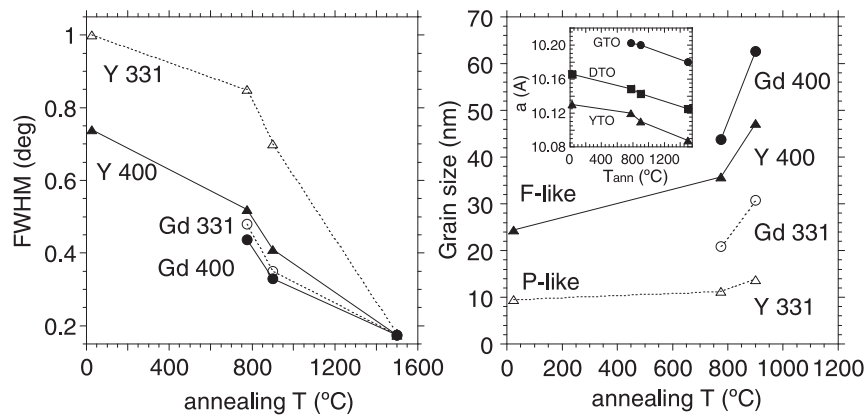

FIG. 2. Comparison of the temperature dependence of the full width of the (331) superstructure peak compared to the fluorite-like (400) reflection (left panel) and the grain sizes estimated from peak broadening (right panel). Errors in grain size are estimated to be $\pm 10 \%$. The inset in the right panel shows the lattice parameters as a function of annealing temperature. Errors in this case are of about $\pm 0.005 \AA$.

and grain sizes determined from peak broadening are shown in Fig. 2 and will be discussed later.

For the three compounds, the pattern approaches that of the reference pyrochlores as the annealing temperature is increased, as a result of improved crystallization. The broadness of the reflections indicates small crystallite size even for the samples annealed at $900{ }^{\circ} \mathrm{C}$. The diffractograms of as-milled YTO and DTO are unambiguously identified as belonging to a cubic pyrochlore structure and superstructure peaks are clearly visible. However, some amount of amorphous material may be present giving rise to a broad background notably under the peak at $2 \theta=30^{\circ}$. This background is absent for samples annealed at or above $775^{\circ} \mathrm{C}$. In contrast for as-milled GTO the proportion of amorphous/crystalline material is reversed, and the main peaks of a crystalline lattice are very faint. Even for 775-MM GTO a weak amorphous background can still be seen at $2 \theta=30^{\circ}$ and as a broad bump between $2 \theta=40^{\circ}$ and $70^{\circ}$. In this respect, it is interesting to note that two different broadening effects are observed: the first one is a general broadening of the diffractogram, affecting both the main, fluorite-like peaks, and the pyrochlore superstructure reflections. It decreases with thermal treatment implying that crystallization is improved upon increasing annealing temperature, a common behavior in compounds synthesized by low-temperature methods. The second is a differential size effect, according to which broader peaks are found for pyrochlore superstructure peaks, even for samples treated at $900^{\circ} \mathrm{C}$. For example, we show in Fig. 2 the full width of the (331) superstructure peak $\left(2 \theta \simeq 38.4^{\circ}\right)$ compared to the fluorite-like (400) reflection $\left(2 \theta \simeq 35.1^{\circ}\right)$ as a function of the annealing temperature, $T_{\text {ann }}$. Both reflections narrow with increasing $\mathrm{T}_{\mathrm{ann}}$, but the (331) peak is always broader than the (400) except for the sample produced by ceramic methods, in which, within error, all peaks have the same width. For YTO (and also for DTO), the differential broadening is remarkable even at $\mathrm{T}_{\mathrm{ann}}=900^{\circ} \mathrm{C}$. For GTO, the trend is the same, but i) reflections are hard to distinguish in the diffractogram of the as-milled sample, and ii) the difference between substructure and superstructure peaks is much smaller. The immediate interpretation of differential broadening is that, upon an underlying fluorite-like structure, 
the pyrochlore-like ordering has a shorter correlation length, so that the system might be described as if formed by pyrochlore domains embedded in a fluorite-like substructure. Estimates of the grain size obtained using the Scherrer formula and taking the SS compounds as a reference for instrumental linewidth (see right panel of Fig. 2), show that the size of pyrochlore-like domains in the MM samples of YTO and DTO, $\sim 10 \mathrm{~nm}$, is about half the size of the fluorite-like regions $(\sim 20-30 \mathrm{~nm})$. In this respect, we note that these values have been determined under the assumption that peak broadening comes exclusively from size effects, i.e., neglecting other contributions such as strain-induced broadening, so that our sizes may be underestimated (see Ref. 7 for a discussion). The trend, however, is clear: both fluorite and pyrochlore-like regions grow with annealing temperature, but fluorite-like domains are always larger than pyrochlore ones. Figure 2 also shows that domains are larger for GTO than for YTO or DTO pyrochlores, indicating that, after crystallization, GTO orders faster than the other titanates.

To shed light on this question it is necessary to use techniques sensitive to the local structure. As commented in Sec. I, we have chosen to this purpose both Raman and X-ray absorption spectroscopies.

\section{A. Raman spectroscopy}

The sensitivity of Raman spectroscopy to oxygen displacements makes it a useful technique in systems where oxygen shifts induce a structural change, as in the fluorite/pyrochlore transition, and also in cases where local atomic ordering results in spatial fluctuations of the structure, which may also occur in disordered pyrochlores.

Because pyrochlore can be seen as a superstructure of fluorite induced by the ordering of $\mathrm{A}$ and $\mathrm{B}$ cations into nonequivalent crystallographic sites and the ordering of anions into three sites $48 f\left(\mathrm{O}_{1}\right), 8 a\left(\mathrm{O}_{2}\right)$, and $8 b\left(\mathrm{O}_{3}\right)$, the latter remaining empty in a perfectly ordered lattice, a brief description of pyrochlore and fluorite Raman activity is relevant. In a perfect $\mathrm{AO}_{2}$ fluorite ( $F m 3 m$ space group, $\mathrm{Z}=4$ ), only one mode $\left(\mathrm{T}_{2 g}\right)$ is expected, which involves oxygen vibration in the tetrahedral cage formed by four $A$ cations. In $\mathrm{A}_{2} \mathrm{~B}_{2} \mathrm{O}_{7}$ pyrochlores $(F d 3 m, \mathrm{Z}=8)$ there are six Raman active modes $\left(\mathrm{A}_{1 g}, \mathrm{E}_{g}, 4 \mathrm{~T}_{2 g}\right)$, involving only vibrations of oxygens at both $48 f$ and $8 a$ sites. A and B cations occupy centrosymmetric sites and therefore they do not contribute to Raman active modes. Typical wavenumbers in $\mathrm{Ti}$ pyrochlores at room temperature are: $\left[\mathrm{A}_{1 g}\right] \sim 520 \mathrm{~cm}^{-1},\left[\mathrm{E}_{g}\right] \sim 330 \mathrm{~cm}^{-1},\left[\mathrm{~T}_{2 g}\right] \sim$ 200, 310, 450, and $580 \mathrm{~cm}^{-1}$ (Refs. 18 and 19). The most characteristic features of Ti-pyrochlore spectra are an intense band at $\sim 320 \mathrm{~cm}^{-1}$, which includes contributions from the $\mathrm{E}_{g}$ mode and from a $\mathrm{T}_{2 g}$ attributed mostly to $\left(\mathrm{O}_{2}\right)-\mathrm{A}-\left(\mathrm{O}_{2}\right)$ bond bending, and the $\mathrm{A}_{1 g}$ band at $520 \mathrm{~cm}^{-1}$, which involves the modulation of the only free parameter of the crystal structure, $\mathrm{x}$, through the vibration of $\mathrm{O}_{1}$ along $\langle 100\rangle$ cubic axes. A peculiarity of the pyrochlore structure is that, according to calculations, ${ }^{20,21}$ none of the Raman active modes involves to any great extent $\mathrm{Ti}-\mathrm{O}$ bond stretching, a vibration that is expected to give the highest wavenumbers since it involves the shortest bonds. In addition to the allowed modes, the spectra of all titanate pyrochlores show additional features, which arise from second-order excitations, crystal field transitions, and positional disorder, if present. ${ }^{19}$

We have performed two series of Raman measurements in the YTO, GTO, and DTO pyrochlores: (i) in situ heating runs, in which spectra are measured as a function of temperature by heating the as-milled (MM) samples at intervals of 50 or $100{ }^{\circ} \mathrm{C}$, letting them equilibrate for $5 \mathrm{~min}$ and then recording the spectrum, and (ii) after-heating measurements, in which we recorded the spectra of the MM samples annealed at 775 and $900{ }^{\circ} \mathrm{C}$, and in polycrystalline samples produced by $\mathrm{SS}$ reaction. The same three series of samples have been used in the XAS experiments reported below.

Figure 3 shows the temperature evolution of the spectra of YTO, GTO, and DTO in in situ experiments. Spectra have been divided by the Bose factor $\left(\mathrm{n}_{B}+1\right)$, where $\mathrm{n}_{B}=$ $[\exp (\hbar \omega / \mathrm{kT})-1]^{-1}$, to suppress the thermally activated lowfrequency background.

The spectra of the as-milled DTO and YTO samples are very similar, and are unambiguously identified as pertaining to a (highly defective) pyrochlore compound. In particular, the characteristic $\mathrm{T}_{2 g}+\mathrm{E}_{g}\left(320 \mathrm{~cm}^{-1}\right)$ and $\mathrm{A}_{1 g}\left(520 \mathrm{~cm}^{-1}\right)$ bands are well defined, with the additional presence of bands at 140 and $600 \mathrm{~cm}^{-1}$ and a very intense broad band extending from 650 to $900 \mathrm{~cm}^{-1}$. Both compounds also show a similar thermal evolution. As temperature increases all features broaden and shift very smoothly until, at a certain temperature between 750 and $800{ }^{\circ} \mathrm{C}, \mathrm{T}^{*}$, the large high-frequency band loses most of its intensity and shifts to a lower frequency. At the same time, the main $\mathrm{T}_{2 g}$ and $\mathrm{A}_{1 g}$ bands are enhanced. The $\mathrm{A}_{1 g}$ mode, directly related to the oxygen $\mathrm{x}$ parameter, hardens abruptly at $\mathrm{T}^{*}$, indicating a steep change of the Ti-O environment.

The spectrum of as-milled GTO is quite different to those of DTO or YTO and consists of just two broad, unstructured bands centered at $\sim 350$ and $750 \mathrm{~cm}^{-1}$, respectively. Upon heating, the spectrum remains almost unchanged up to $700^{\circ} \mathrm{C}$. Then, at some temperature between 700 and $800^{\circ} \mathrm{C}, \mathrm{T}_{1}$, the pyrochlore spectrum appears quite suddenly, indicating the crystallization of a pyrochlore structure at a spatial extent enough to activate the characteristic Raman spectrum, typically about $5 \mathrm{~nm}$. Indeed, the spectrum at $800{ }^{\circ} \mathrm{C}$ resembles that of the as-milled YTO and DTO. On further heating, the evolution is parallel to that of YTO and DTO: at $\mathrm{T}^{*} \sim 900^{\circ} \mathrm{C}$ pyrochlore bands are enhanced and the high frequency band decreases and softens significantly. Quite interestingly, the high frequency band does not disappear at the temperature of formation of pyrochlore lattice $\left(\mathrm{T}_{1}\right)$ suggesting that the short bonds responsible for that band are still present in the just crystallized pyrochlore, as in the spectrum of as-milled YTO and DTO. After cooling down to RT (spectra not shown), the pyrochlore bands appear narrower than in the as-milled compounds but not as narrow as in the reference SS samples. Moreover, some extra bands are still present at high and low frequency, implying that a totally ordered distribution has not been achieved.

Figure 4 shows the spectra of four different samples of each compound: as-milled, thermally treated 775-MM and 900-MM samples and the reference SS samples. As in the in-situ experiments, YTO and DTO behave similarly. As described above, the as-milled materials unambiguously show 


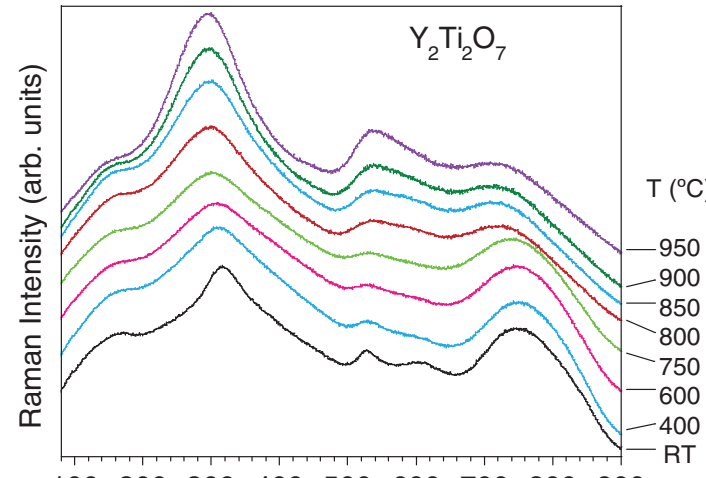

100200300400500600700800900 Raman shift $\left(\mathrm{cm}^{-1}\right)$
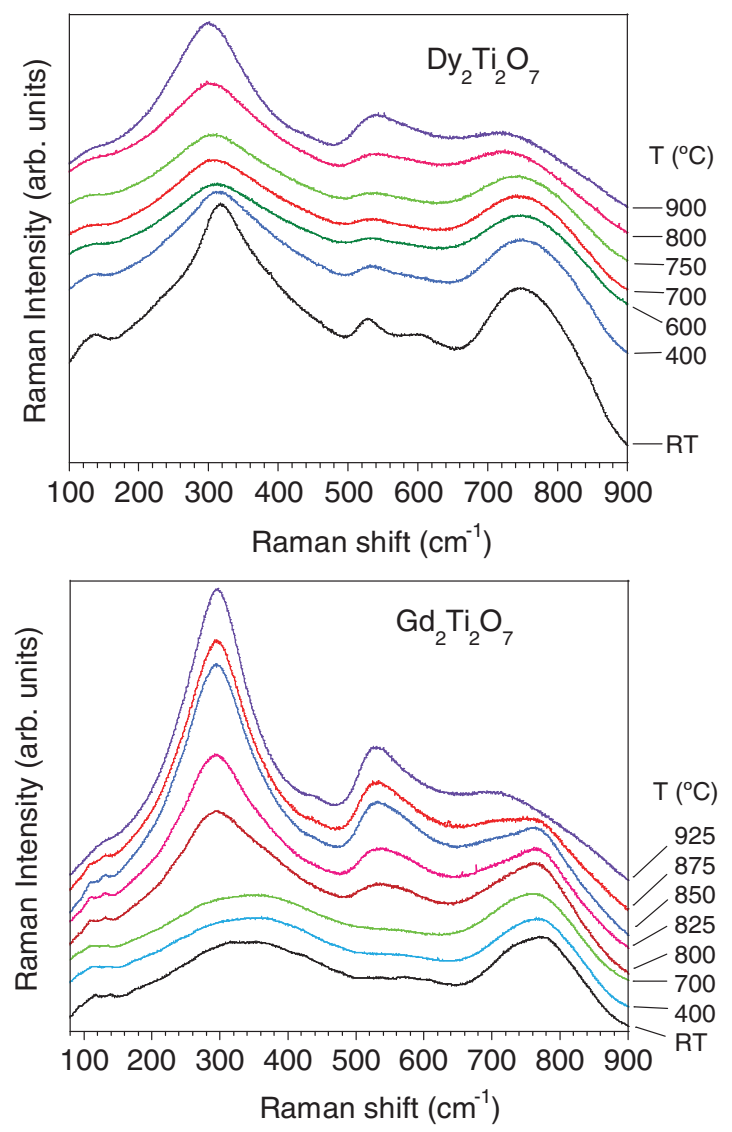

FIG. 3. (Color online) Temperature evolution of the Raman spectra of $\mathrm{Y}_{2} \mathrm{Ti}_{2} \mathrm{O}_{7}, \mathrm{Dy}_{2} \mathrm{Ti}_{2} \mathrm{O}_{7}$, and $\mathrm{Gd}_{2} \mathrm{Ti}_{2} \mathrm{O}_{7}$. Spectra have been divided by the Bose factor $\left(\mathrm{n}_{B}+1\right)$, where $\mathrm{n}_{B}=[\exp (\hbar \omega / \mathrm{kT})-1]^{-1}$.

the main bands of pyrochlores, with some additional features at 140 and $600 \mathrm{~cm}^{-1}$, and a large high frequency band centered at around $750 \mathrm{~cm}^{-1}$. All bands are broad and, on the whole, the spectra are about one order of magnitude less intense than in the SS reference samples.

Samples annealed at $775^{\circ} \mathrm{C}$ show the same features as the MM samples, although considerably sharpened. The intensities of the pyrochlore-like bands have increased relative to that of the forbidden, disorder-induced bands. The most noticeable change is the significant decrease of the high frequency band and its shift toward lower frequencies (inset, Fig. 5). The trend is qualitatively the same in the samples
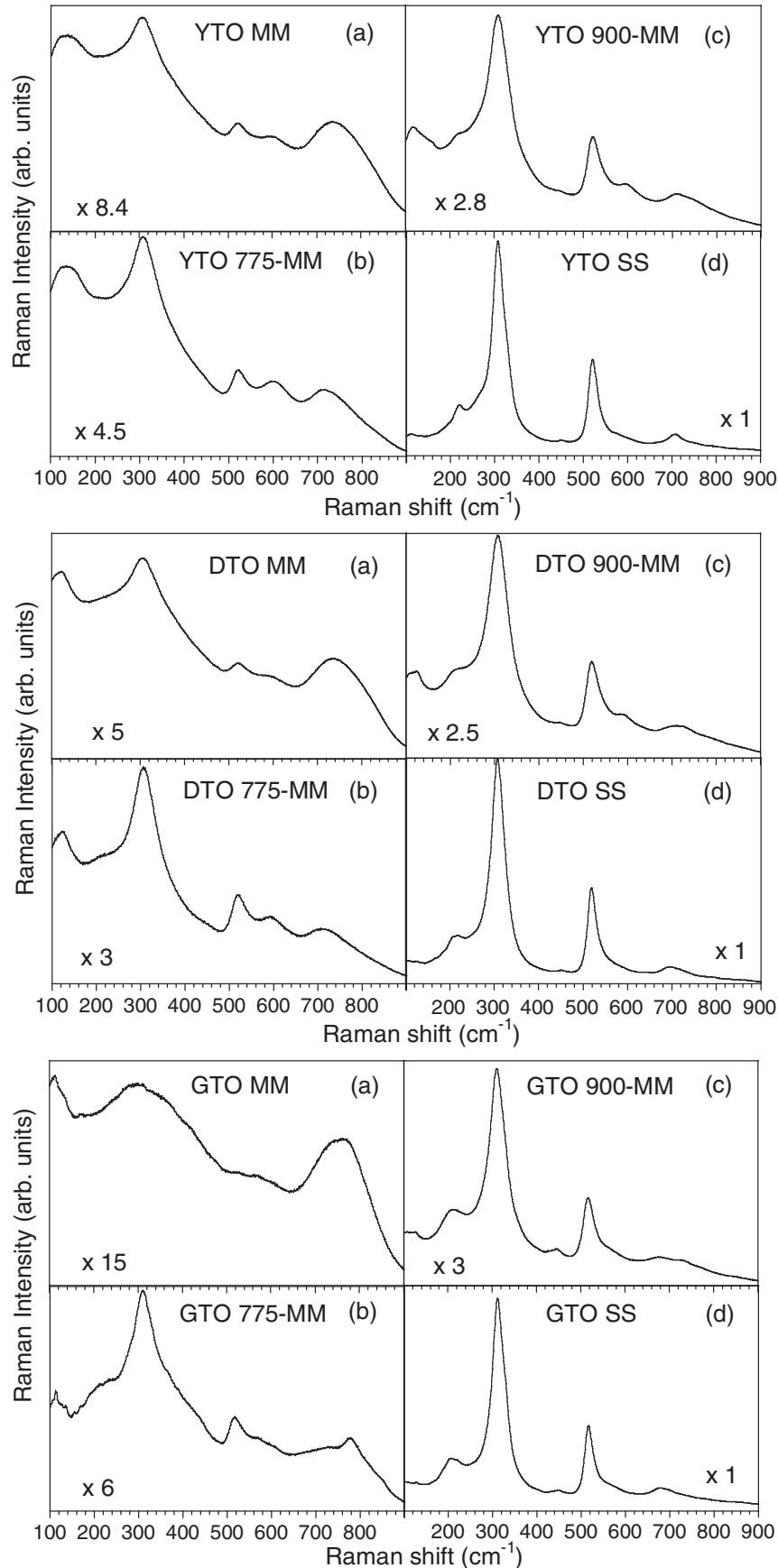

FIG. 4. Raman spectra of $\mathrm{MM} \mathrm{Y}_{2} \mathrm{Ti}_{2} \mathrm{O}_{7}, \mathrm{Dy}_{2} \mathrm{Ti}_{2} \mathrm{O}_{7}$, and $\mathrm{Gd}_{2} \mathrm{Ti}_{2} \mathrm{O}_{7}$ pyrochlores recorded at $\mathrm{RT}$ as a function of thermal treatment. For each compound we show spectra of as-milled material (a), samples annealed at $775{ }^{\circ} \mathrm{C}(\mathrm{b}), 900^{\circ} \mathrm{C}$ (c), and reference samples produced by SS reaction method (d).

annealed at $900{ }^{\circ} \mathrm{C}$. The spectra now resemble the respective $\mathrm{SS}$ references, though weak forbidden bands remain. The highfrequency band appears considerably shifted toward lower wavenumbers.

GTO behaves in a substantially different way. As explained before, the as-milled material does not present pyrochlore like features, in agreement with the quasi-amorphous x-ray diffractogram (Fig. 1). Interestingly, it shows a high-frequency band similar to that of DTO and YTO, though shifted to higher 
frequencies. At $775^{\circ} \mathrm{C}$ pyrochlore bands have appeared, but they seem to be superposed on an amorphous background, which suggests that crystallization is still incomplete at this temperature. The spectrum of the sample annealed at $900{ }^{\circ} \mathrm{C}$, however, indicates a higher order in GTO compared to DTO and YTO at the same temperature and closely resembles that of SS material, except for some residual broadening and weak high-frequency bands.

$\mathrm{A}_{2} \mathrm{~B}_{2} \mathrm{O}_{7}$ pyrochlores are characterized by the ordering of $\mathrm{A}$ and $\mathrm{B}$ cations in two different sites and the separation of oxide anions into two types: $\mathrm{O}_{1}$ in $48 f$ site and $\mathrm{O}_{2}$ at $8 a$ site, with a third anion site $\left(\mathrm{O}_{3}, 8 b\right)$ remaining empty. Over that perfect ordering, local defects may occur, the most common being cation antisite defects and Frenkel pairs consisting of an oxygen vacancy in either the $\mathrm{O}_{1}$ or $\mathrm{O}_{2}$ sublattices close to an interstitial oxygen, normally occupying the $\mathrm{O}_{3} 8 b$ site. Depending on the nature and relative size of $\mathrm{A}$ and $\mathrm{B}$ cations, the pyrochlore lattice can afford a varying degree of atomic positional disorder before transforming to either a defect fluorite structure, if $\mathrm{r}_{A} \gtrsim \mathrm{r}_{B}$, or to an amorphous state, when $\mathrm{r}_{A} \gg \mathrm{r}_{B}$. YTO and DTO belong to the first class, and can afford a large amount of disorder while preserving the pyrochlore structure, whereas GTO readily transforms under heavy ion irradiation or high pressure to an amorphous state. ${ }^{22-24}$ High energy processes involved in mechanical milling are expected to yield similar effects and in fact, as-grown products of mechanical milling are usually highly disordered; thermal treatments may relax internal stresses until equilibrium distribution is achieved. The presence of short- or medium-range ordered regions is thus to be expected as a typical feature of mechanically milled systems.

As explained above, the spectra of as-milled YTO and DTO can be unambiguously attributed to a pyrochlore, implying that large enough regions with distinct population of $\mathrm{A}$ and $\mathrm{B}$ cation sites and of $\mathrm{O}_{1}, \mathrm{O}_{2}$, and $\mathrm{O}_{3}$ oxygen sites are present in these samples. The spectra, however, indicate a high degree of disorder. Both cations and anions are likely involved in this disorder, in the form of cation antisite defects together with oxygen vacancies and a non-vanishing occupancy of the usually vacant $\mathrm{O}_{3}$ sites. In contrast with DTO and YTO, the spectrum of as-milled GTO (Fig. 4) cannot be identified as that of a pyrochlore. Similar spectra found in the literature have been attributed to amorphization induced either by high pressure $^{22}$ or ion irradiation, ${ }^{23,24}$ which is in agreement with the quasi amorphous aspect of the x-ray diffractogram of the as-milled GTO material (Fig. 1).

The most conspicuous characteristic of the spectrum of as-milled samples is the large high frequency band centered at $\sim 750 \mathrm{~cm}^{-1}$. Its high frequency suggests it in some way involves Ti-O bond stretching and $\mathrm{O}-\mathrm{Ti}-\mathrm{O}$ angle bending, which according to the calculations of Gupta et al. ${ }^{20,21}$ are the internal coordinates with the highest force constants in Ti pyrochlores. A decomposition of the band using lorentzian profiles (Fig. 5), shows that it contains at least three components, at $\sim 725,780$, and $820 \mathrm{~cm}^{-1}$, with small differences among the three compounds. In a simple two-atom model, which relates the frequency of vibration to the length of the bond involved in the vibration, different bands would be related to different $\mathrm{Ti}-\mathrm{O}$ bond lengths, but they may also be indicative of different titanium environment or vibrational patterns. As

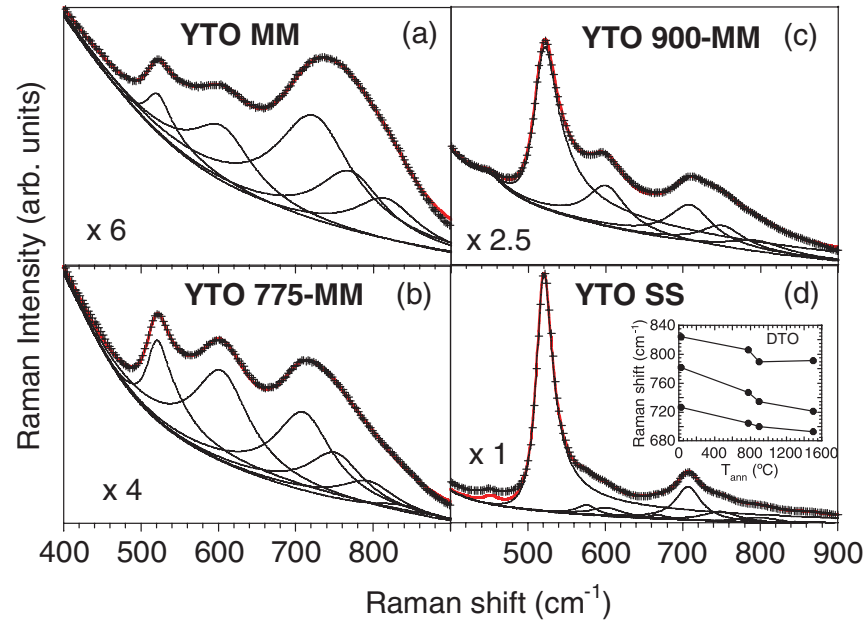

FIG. 5. (Color online) Decomposition of the spectrum of YTO in the $500-900 \mathrm{~cm}^{-1}$ region as superposition of lorentzian profiles, to put into evidence the presence of several components in the high frequency band as well as its evolution upon thermal annealing. The experimental spectra (+) are compared to the result of the fitting (red, solid line), with a background added to account for features appearing at lower frequencies.

temperature increases the second and third subbands decrease compared to the first subband. On the whole, all three components decrease with respect to the allowed spectrum. In the usual spectrum of $\mathrm{Ti}$ pyrochlores (see reference $\mathrm{SS}$ spectra), the band at $\sim 700 \mathrm{~cm}^{-1}$ is attributed to second order excitations. ${ }^{19}$

The high-frequency (hf) band has been observed in other Ti containing pyrochlores and has been interpreted in different ways. Glerup et al. ${ }^{25}$ attribute the appearance of this band in $\mathrm{Y}_{2}(\mathrm{Ti}, \mathrm{Zr})_{2} \mathrm{O}_{7}$ and in Ti-doped $\mathrm{YSZ}_{\text {to }} \mathrm{TiO}_{7}$ clusters. This assumption is mainly based on the fact that this band increases with $\mathrm{Zr}$ content in $\mathrm{Ti} / \mathrm{Zr}$ mixed systems as the structure evolves toward a more disordered lattice, eventually reaching a fluorite-like one in which the average cation coordination is sevenfold. The band is also observed in $\mathrm{Gd}_{2}(\mathrm{Ti}, \mathrm{Zr})_{2} \mathrm{O}_{7}$ solid solutions at intermediate $\mathrm{Ti}$ content, ${ }^{22,26}$ i.e., still in the pyrochlore domain but with a high degree of cation and anion disorder. Alternatively, Zhang and Saxena ${ }^{27}$ interpret the high-frequency band in amorphisized GTO as resulting from non correlated $\mathrm{TiO}_{6}$ vibrations, with the argument that the basic units of GTO are $\mathrm{TiO}_{6}$ octahedra and that these are preserved even in the amorphous state.

Although the hf band is with no doubt related to disorderactivated oxygen vibration, its interpretation is far from being clear. The occurrence of sevenfold coordination for $\mathrm{Ti}$, as proposed by Glerup et al., ${ }^{25}$ is unlikely. Though it is true that the cation coordination number $(\mathrm{CN})$ in $(\mathrm{A}, \mathrm{B})_{4} \mathrm{O}_{7}$ defect fluorites is 7 , this is only an average. In practice, each cation will tend to retain its own $\mathrm{CN}$ : 8 for large $\mathrm{A}^{3+}$ cations and 6 for small $\mathrm{B}$ cations as $\mathrm{Ti}^{4+} . \mathrm{Zr}^{4+}$ is a special case, because, although it nominally enters the $\mathrm{B}$ site, its size is close to that of rare earths, so that it may easily adopt $\mathrm{CN}=7$. Then, in $\mathrm{Ti} / \mathrm{Zr}$ pyrochlore solid solutions, with $\mathrm{Ti}$ and $\mathrm{Zr}$ nominally sharing the $\mathrm{B}$ site, it is likely that $\mathrm{Zr}$ increases its $\mathrm{CN}$ either by forcing an extra oxygen ion to occupy a next-neighbor $8 b$ 
site or by exchanging sites with $\mathrm{Gd}$, which in turn will also tend to increase its $\mathrm{CN}$ at the $\mathrm{B}$ site. Any of these possibilities will result on average in a lower coordination for $\mathrm{Ti}$ at the $\mathrm{B}$ site in the form of $5 \times \mathrm{O}_{1}$ or $5 \times \mathrm{O}_{1}+\mathrm{O}_{3}$ configurations, together with the usual $6 \mathrm{O}_{1}$ octahedral ones. In our case, only one type of $\mathrm{B}$ cation exists, but milling may produce a high density of cation antisite defects. As a result, we attribute the high-frequency band in YTO, DTO, and GTO to the vibration of oxygen atoms bonded to $\mathrm{Ti}^{4+}$ ions in low coordination $(5$ or $5+1)$, resulting from the presence of cation antisite defects and subsequent oxygen sublattice rearrangement. As the coordination number is reduced, the $\mathrm{Ti}-\mathrm{O}$ bond distance is expected to decrease and yield relatively high frequency bands, as observed.

The results of Glerup et $a{ }^{25}$ can be explained within this model. The main argument of Glerup et al. is that $\mathrm{Ti}$ would adopt $\mathrm{CN}=7$ when entering a fluorite lattice, as in YSZ or in highly disordered pyrochlores. In fact, the band appears in $\mathrm{Y}_{2}(\mathrm{Ti}, \mathrm{Zr})_{2} \mathrm{O}_{7}$ solid solutions not only in fully disordered systems with defect-fluorite (DF) structure, but also at intermediate $\mathrm{Zr}$ concentrations, where the long-range structure is still that of a pyrochlore, and thus Ti is likely to preferentially occupy the sixfold coordinated $\mathrm{B}$ site. Moreover, all compositions in Ref. 25, from $\mathrm{Y}_{2} \mathrm{Ti}_{2} \mathrm{O}_{7}$ to $\mathrm{Y}_{2} \mathrm{TiZrO}_{7}$, are pyrochlores with no cation antisite defects, i.e., with $\mathrm{Ti}$ and $\mathrm{Zr}$ sharing the $\mathrm{B}$ site, but the occupancy of the $\mathrm{O}_{3}$ site increases as $\mathrm{Zr}$ is introduced. This occurs at the expense of the $\mathrm{O}_{1} 48 \mathrm{f}$ oxygen ions, since the $\mathrm{O}_{2}$ sublattice remains fully occupied. It seems evident that the occupancy of vacant sites tends to satisfy a higher $\mathrm{CN}$ for $\mathrm{Zr}$ in the $\mathrm{B}$ site, thus a lower $\mathrm{CN}$ for $\mathrm{Ti}$ in the same site. Similar arguments are derived from the neutron diffraction studies of $\mathrm{Y}_{2}(\mathrm{Ti}, \mathrm{Zr})_{2} \mathrm{O}_{7}$ by Heremans et al., ${ }^{28}$ who conclude that in a first step of pyrochlore disordering there is no A-B exchange and that, at this stage, only the $\mathrm{O}_{1}$ sublattice is affected by disorder, thus reinforcing the idea that vacancies are first formed in the $48 f$ sublattice, and that this will increase (decrease) the coordination of large (small) cations at B site, respectively.

Comparison with other Ti oxides also provides arguments in favor of low ( 5 or 6 ) $\mathrm{CN}$ for $\mathrm{Ti}$. In general, frequencies above $750 \mathrm{~cm}^{-1}$ indicate short Ti-O distances $(\sim 1.8 \AA)$ and $\mathrm{CN}=$ 4 or 5 , whereas the Raman spectrum of oxides containing quasi-octahedral $\mathrm{TiO}_{6}$ units usually does not extend beyond $600 \mathrm{~cm}^{-1}$. So, in $\mathrm{Sr}_{2} \mathrm{TiO}_{4}$ (Ref. 29), with the tetragonal $\mathrm{K}_{2} \mathrm{NiF}_{4}$ structure, the highest allowed Raman band appears at $578 \mathrm{~cm}^{-1}$ and is attributed to the vibration of the apical oxide ions bonded to hexa-coordinated Ti with $\mathrm{d}\left(\mathrm{Ti}-\mathrm{O}_{\mathrm{ap}}\right)=1.915 \AA$. In $\mathrm{La}_{(2-x) / 3} \mathrm{Li}_{x} \mathrm{TiO}_{3}$ perovskites, with corner-sharing $\mathrm{TiO}_{6}$ octahedra and average $\mathrm{d}_{\mathrm{Ti}-\mathrm{O}} \sim 1.93 \AA$, a disorder-enhanced band, attributed to local perturbation at oxygen sites by nearby lithium atoms, is found at frequencies extending from 550 to $590 \mathrm{~cm}^{-1}$. (Ref. 30) However, in Ti oxides with pentacoordinated $\mathrm{Ti}$, such as $\mathrm{La}_{2} \mathrm{TiO}_{5}$ and $\mathrm{Nd}_{2} \mathrm{TiO}_{5}$, the Raman spectrum presents a band at $770 \mathrm{~cm}^{-1}$ which is attributed to Ti-O stretching vibration. ${ }^{22,31}$ It is interesting to compare our results with the XANES data on $\mathrm{Ca}(\mathrm{Zr}, \mathrm{Ti}) \mathrm{O}_{3}$ perovskites, ${ }^{32}$ where $\mathrm{Ti}$ and $\mathrm{Zr}$ nominally occupy octahedral sites. $\mathrm{Xu}$ et al. show $^{32}$ that, for samples produced by sol-gel methods and treated at temperatures below $500{ }^{\circ} \mathrm{C}$, the coordination number of $\mathrm{Ti}$ was lower than 6 , with a tendency to increase upon thermal treatment. Coordination numbers higher than 6 where simultaneously found for $\mathrm{Zr}$ in the same samples.

We can get more insight into the origin of the hf band by comparing the evolution of our MM samples upon increasing temperature with the trend of increasing order found in $\mathrm{A}_{2}(\mathrm{Ti}, \mathrm{Zr})_{2} \mathrm{O}_{7}$ solid solutions on increasing $\mathrm{Ti}$ content. In a fluorite, cations are in the center of a cube of oxygen atoms so that $\mathrm{CN}=8$. In ordered $\mathrm{A}_{2} \mathrm{~B}_{2} \mathrm{O}_{7}$ pyrochlores the A cation keeps $\mathrm{CN}=8$, though the eight oxygen atoms do not form a cube but a scalenohedron of $2 \times \mathrm{O}_{2}+6 \times \mathrm{O}_{1}$, whereas the $\mathrm{B}$ cation adopts a pseudo-octahedral coordination formed by $6 \times \mathrm{O}_{1}$. A highly disordered pyrochlore may eventually reach a $(\mathrm{A}, \mathrm{B})_{4} \mathrm{O}_{7}$ defect fluorite structure with cation $\mathrm{CN}=7$ on average. It is experimentally observed in $\mathrm{A}_{2}(\mathrm{Ti}, \mathrm{Zr})_{2} \mathrm{O}_{7}$ solid solutions that increasing atomic disorder is accompanied by the increase of average $\mathrm{B}-\mathrm{O}$ bond distance, due to the substitution of larger $\mathrm{Zr}$ or rare earth cations into the $\mathrm{B}$ site. A long $\mathrm{Ti}-\mathrm{O}$ bond would result in a low frequency of vibration which is difficult to reconcile with the appearance of a band centered at $750 \mathrm{~cm}^{-1}$. Conversely, if on ordering the Ti coordination were to change from 7 to 6 , the Ti-O distance would decrease, resulting in a higher frequency, contrary to the experimental observation, which is more indicative of an increase of the Ti-O bond length at $\mathrm{T}^{*}$. Thus, we conclude that $\mathrm{Ti}_{B}-\mathrm{O}$ and $\mathrm{R}_{B}-\mathrm{O}$ bonds behave differently, which leads again to the model based on preferential $\mathrm{CN}$ of each cation type. ${ }^{33}$

In summary, according to our Raman results the change of the Ti coordination at $\mathrm{T}^{*}$ seems to be opposite to that expected on ordering from a defective fluorite to pyrochlore: instead of a reduction of the $\mathrm{CN}$ from 7 to 6 , the downshift of the high-frequency band suggests an increase of the Ti CN from 5 $($ or $5+1)$ to 6 .

Though it is clear that the process undergone by the mechanically milled pyrochlores upon heating is that of ordering from a highly disordered or defective pyrochlore toward a well ordered structure, the details of the process are not fully understood. The fact that the Raman spectrum remains basically constant below $\mathrm{T}^{*}$ suggests that the process is a thermally activated one, very likely driven by ion diffusion. However, it is difficult to ascertain, only from Raman results, whether the ordering is driven by cations, anions, or both at the same time. There is a long standing discussion of which one determines the other, i.e., whether cations or anions order first, and different techniques seem to provide contradictory results regarding this issue, ${ }^{28,34-37}$ although the most accepted model is that cation and anionic disorder go together. A different question arises as which is the best description of atomic distribution in disordered pyrochlores and whether disorder is due to point defect concentration or to size effects. There are basically two possibilities: the first one is that of a lattice preserving the long-range pyrochlore structure but with a high density of defects, notably cation antisite and anion Frenkel pairs, eventually leading to defect fluorite structure. An alternative description is based on the presence of ordered pyrochlore domains with antiphase domain boundaries among them, where $\mathrm{A}$ and $\mathrm{B}$ cations exchange sites and oxygen atoms become disordered. The domain model has been proposed to explain results on $\mathrm{A}_{2} \mathrm{Zr}_{2} \mathrm{O}_{7}$ compounds from several techniques: the similarity of the Raman spectrum of $\mathrm{Gd}_{2} \mathrm{Zr}_{2} \mathrm{O}_{7}$ with either pyrochlore or fluorite structure; ${ }^{38}$ the presence 
of anti phase domain boundaries in electron microscopy of $\mathrm{A}_{2} \mathrm{Zr}_{2} \mathrm{O}_{7}$ compounds with a fluorite diffractogram; ${ }^{39}$ and the finding by EXAFS that the coordination numbers of $\mathrm{Gd}$ and $\mathrm{Zr}$ in $\mathrm{Gd}_{2} \mathrm{Zr}_{2} \mathrm{O}_{7}$ are 8 and 6 , respectively, irrespective of whether the long-range structure is either pyrochlore or fluorite. ${ }^{40}$

Unfortunately, it is not possible to unambiguously conclude which of the above models is best to account for the Raman spectrum of our samples. In contrast to $\mathrm{Zr}$ pyrochlores, titanates do not readily transform to a defect fluorite structure upon disordering. Mechanical milling, however, provides the high energy required to produce a high density of cation and anion defects, yielding a situation close to total disorder. The fact that the X-ray diffractogram of YTO and DTO as-milled samples is already pyrochlore-like implies that long-range pyrochlore ordering exists, but differential broadening of fluorite and pyrochlore-like peaks (Fig. 2) suggests that pyrochlore ordering occurs at a shorter length scale within the fluorite lattice, supporting the domain-based model. On the other hand, the broad aspect of the Raman spectrum and the presence of forbidden bands indicate that there is considerable atomic disorder. The narrowing of x-ray reflections and of Raman bands, as well as the enhancement of pyrochlore spectral features upon thermal treatments, suggest that crystallite size increases upon heating and that local defects tend to disappear.

A mixed picture may apply, by which the as-milled material is already a pyrochlore but affords a high defect concentration that results in nanometer-sized pyrochlore-like areas. Upon thermal treatment the system changes to a situation closer to the second model, in which antisite and Frenkel defect concentration decrease rapidly on approaching the ordering event at $\mathrm{T}^{*}$. As a result, the pyrochlore-like domains enlarge through a nucleation and growth phenomenon. This process is thermally activated and the "event" observed in DTA ${ }^{7}$ can be associated to a transition from medium-range to long-range ordering of both cations and anions, typically from tens to hundreds of $\mathrm{nm}$. In GTO there is a previous crystallization process at $\mathrm{T}_{1}$, between 700 and $800{ }^{\circ} \mathrm{C}$. Above that temperature ordering proceeds qualitatively in much the same manner as in DTO or YTO, though at a faster rate: the spectrum of GTO annealed at $900{ }^{\circ} \mathrm{C}$ is closer to that of the reference SS sample than those of DTO and YTO pyrochlores treated at the same temperature. Crystallite sizes determined from XRD are also larger for annealed GTO than for DTO or YTO.

Aimed of verifying these propositions we have extended our study by using x-ray absorption spectroscopy. In this way it should be possible to gain a deeper insight on the local structure, including the coordination number or the presence of vacancies, of both the rare-earth and $\mathrm{Ti}$ atoms. In addition, XAS will allow us to determine both the evolution of the structural disorder and the existence of both oxygen Frenkel pairs and cation antisite defects.

\section{B. X-ray absorption spectroscopy}

The Ti $K$-edge XANES spectra of the $\mathrm{Y}_{2} \mathrm{Ti}_{2} \mathrm{O}_{7}, \mathrm{Gd}_{2} \mathrm{Ti}_{2} \mathrm{O}_{7}$, and $\mathrm{Dy}_{2} \mathrm{Ti}_{2} \mathrm{O}_{7} \mathrm{MM}$ pyrochlores are shown in Fig. 6. The spectra of the as-milled and fired MM samples are compared to those of the samples obtained by traditional SS methods.
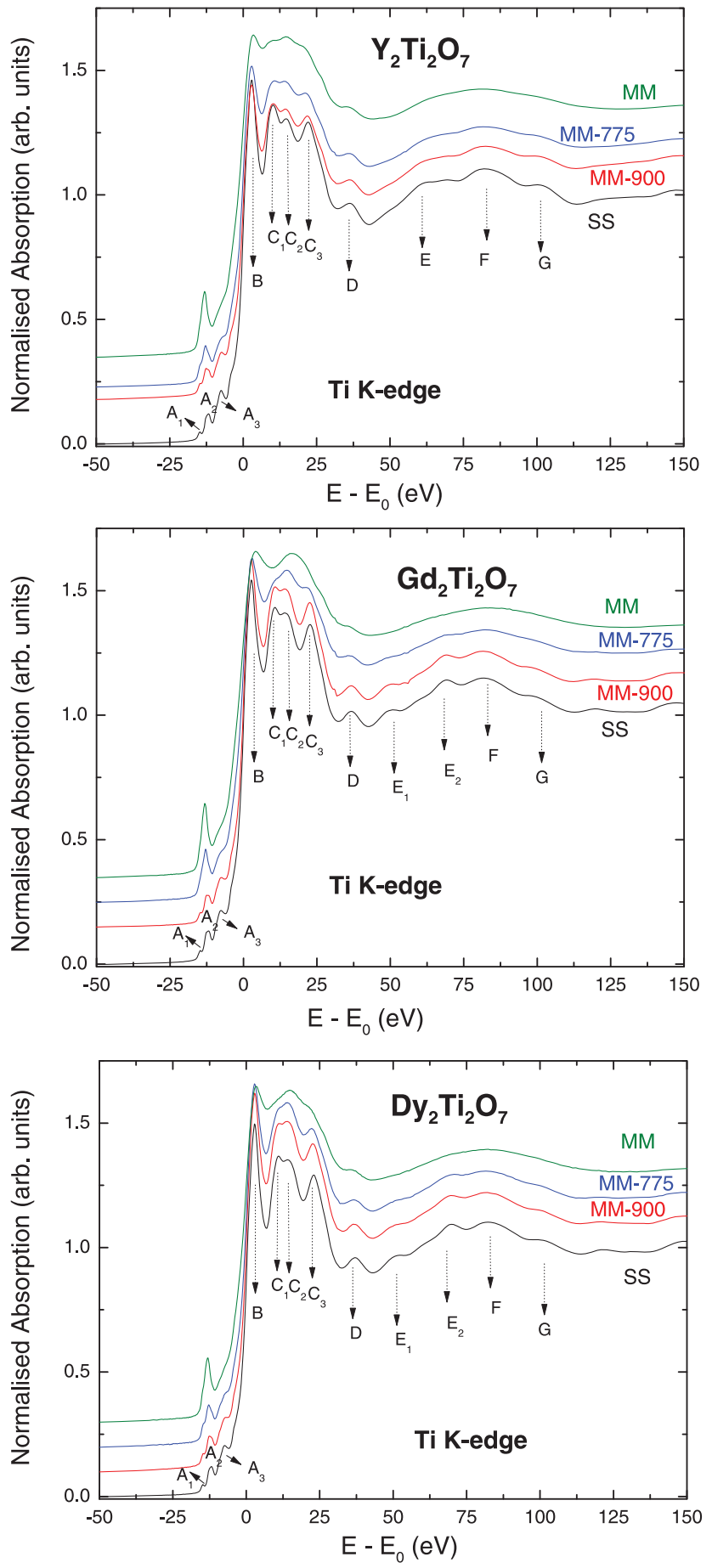

FIG. 6. (Color online) Comparison of the experimental Ti $K$ edge XANES spectra in the case of the as-milled MM samples, MM samples fired at $\mathrm{T}=775^{\circ} \mathrm{C}$ and $900^{\circ} \mathrm{C}$, and the SS samples obtained by traditional methods: $\mathrm{Y}_{2} \mathrm{Ti}_{2} \mathrm{O}_{7}$ (top panel), $\mathrm{Gd}_{2} \mathrm{Ti}_{2} \mathrm{O}_{7}$ (middle), and $\mathrm{Dy}_{2} \mathrm{Ti}_{2} \mathrm{O}_{7}$ (bottom).

In the case of the SS samples, the Ti $K$-edge spectra of the three compounds show a similar spectral profile (we refer its description to the energy scale of $\mathrm{Y}_{2} \mathrm{Ti}_{2} \mathrm{O}_{7}$ ). The spectra are characterized in all cases by the existence of three peaks below the absorption edge (hereafter pre-peaks) at energies $-15 \mathrm{eV}$ $\left(\mathrm{A}_{1}\right),-12 \mathrm{eV}\left(\mathrm{A}_{2}\right)$, and $-7.6 \mathrm{eV}\left(\mathrm{A}_{3}\right)$. In addition, the main 
absorption line exhibits a narrow peak (B) at $\sim 2.8 \mathrm{eV}$ above the absorption edge. Close to this there is a broad resonance showing three well defined peaks $\left(\mathrm{C}_{1}, \mathrm{C}_{2}\right.$, and $\left.\mathrm{C}_{3}\right)$ at $\sim 10.2 \mathrm{eV}$, $15 \mathrm{eV}$, and $22 \mathrm{eV}$ above the edge. For higher energies all the compounds show a peak (D) at $\sim 36.4 \mathrm{eV}$ and another broad resonance in which different spectral features are defined: a wide feature $\mathrm{E}$ at $\sim 61.5 \mathrm{eV}$, and two peaks, $\mathrm{F}$ and $\mathrm{G}$, at $\sim 82.3 \mathrm{eV}$, and $\sim 100.5 \mathrm{eV}$, respectively. In the case of both $\mathrm{Gd}_{2} \mathrm{Ti}_{2} \mathrm{O}_{7}$ and $\mathrm{Dy}_{2} \mathrm{Ti}_{2} \mathrm{O}_{7}$ compounds the peak $\mathrm{E}$ splits in two ones, $\mathrm{E}_{1}$ and $\mathrm{E}_{2}$, located at $\sim 50$ and $\sim 68 \mathrm{eV}$, respectively.

The XANES spectra of the MM samples show a clear departure from those of the SS samples. However, as the compounds are fired at higher temperatures there is a continuous convergence to the SS XANES. In principle, one can suspect that the effect of disorder is very large in the case of the as-milled samples and that as the compounds are annealed the long-range crystalline order sets on. Indeed, the XANES spectra of both the SS and the MM samples fired at $900{ }^{\circ} \mathrm{C}$ show the same spectral features and the slight differences observed can be addressed to structural disorder effects. However, a closer inspection of the spectra indicates that the degree of the structural disorder is different for the three studied materials. Both the as-milled YTO and DTO samples exhibit a greater order than the GTO one. Moreover, the spectrum of the GTO sample fired at $775^{\circ} \mathrm{C}$ is close to those of the as-milled $\mathrm{Y}$ and $\mathrm{Gd}$ samples. These results indicate that the local ordering around the Ti centres evolves differently in the studied pyrochlores. The posed question is, however, to what extent the local structure in the as-milled samples varies with respect to that of reference pyrochlores, due to the disorder effects inherent to the milling process. In this respect, the inspection of the absorption pre-peaks is quite instructive. In the case of both MM - 900 and SS samples, the Ti $K$-edge XANES spectra exhibit three well defined pre-peaks, in agreement with the expected behavior for a sixfold Ti site. ${ }^{15,41}$ In contrast, the spectra of the as-milled MM samples show only a main pre-peak which suggests a lower, four-fold or five-fold, Ti coordination. ${ }^{41}$ It should, however, be noted that the $A_{3}$ pre-peak is close to the absorption threshold and its presence can be obscured by the structural disorder as the edge position depends as the bond lengths. ${ }^{14}$ Indeed, by following the evolution of the $A_{3}$ peak from SS to MM samples it is observed that this peak is progressively less defined and it seems to overlap with the absorption edge. Therefore, no direct conclusion can be derived from the simple fingerprint analysis of XANES regarding the structural order of $\mathrm{Ti}$ atoms in the MM samples.

Trying to get a deeper insight on this subject we have performed the $a b$ initio computation of the Ti $K$-edge XANES. Our main aim is to determine the next-neighbouring Ti-O environment in both MM and SS samples. In particular, we aim to discern whether the smoothed XANES spectra observed in the MM samples in comparison to those of the SS samples is due to that: (i) MM and SS samples exhibit a different Ti-O coordination polyhedron, or (ii) both compounds show the same Ti-O coordination (same $\mathrm{CN}$ and bonding geometry) although with a different degree of structural disorder. If this is the case, multiple scattering processes involving higher coordination shells do not contribute to the XANES. Therefore we should determine which is the short-range Ti arrangement

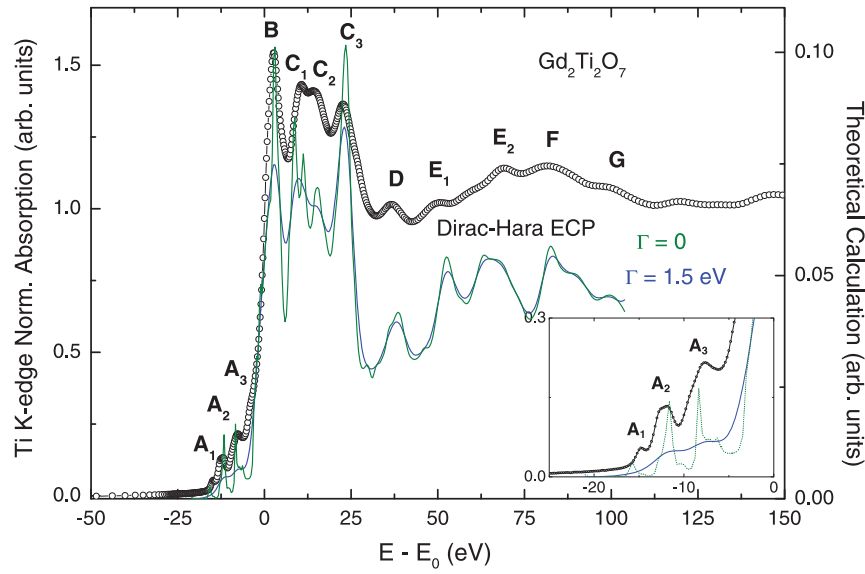

FIG. 7. (Color online) Comparison of the experimental Ti $K$-edge XANES spectrum of $\mathrm{Gd}_{2} \mathrm{Ti}_{2} \mathrm{O}_{7}(\mathrm{O})$ and the results of the calculations performed by using the DH ECP potential (solid line). The dotted lines correspond to the convolution of the theoretical spectra with a Lorentzian shape function to account for the core-hole lifetime.

that is effectively contributing to the XANES spectra. In a second step the study will be extended to the A cations (Y, Gd, and Dy) with the aim of determining if disorder affects equally both $\mathrm{A}$ and $\mathrm{B}$ sites in the $\mathrm{A}_{2} \mathrm{~B}_{2} \mathrm{O}_{7}$ pyrochlores. Finally, these results should be compared to the results of the EXAFS fitting procedure.

$A b$ initio computation of the Ti $K$-edge XANES was initially performed for $\mathrm{Gd}_{2} \mathrm{Ti}_{2} \mathrm{O}_{7}$. We have built a cluster of 171 atoms, i.e., including contributions from neighbours within the first $8 \AA$ around the photoabsorbing $\mathrm{Ti}$ atom (Fig. 7). The computations have been performed by considering the ideal structure of pyrochlores, i.e., without considering either the presence of vacancies or the occupancy of $8 b$ sites. Through these computations we have tested the performance of different exchange and correlation potentials (ECP) to account for the experimental spectrum. ${ }^{13,14}$ The complete set of results are shown in the Fig. 14 of the supplementary material.

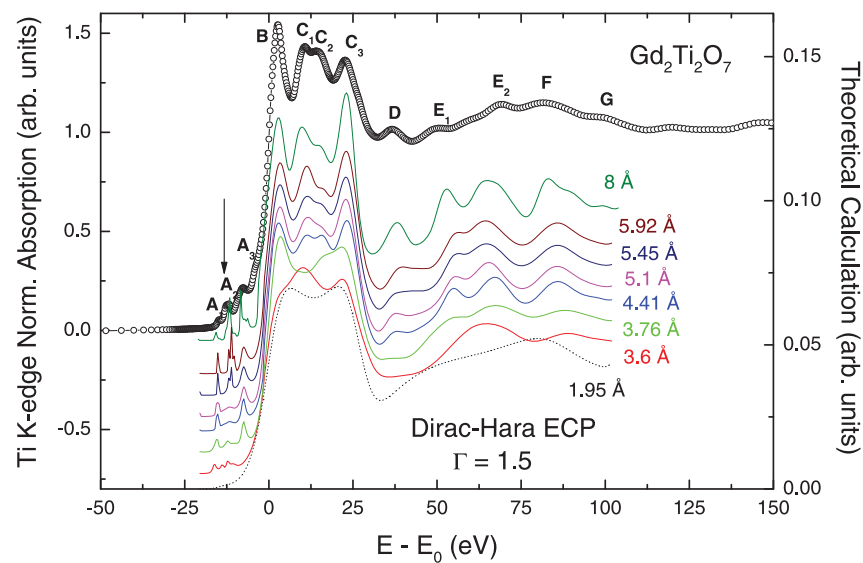

FIG. 8. (Color online) Comparison of the experimental Ti $K$-edge XANES spectrum of $\mathrm{SS} \mathrm{Gd}_{2} \mathrm{Ti}_{2} \mathrm{O}_{7}$ (०) and the results of the calculations performed using the DH ECP of clusters of different size. 

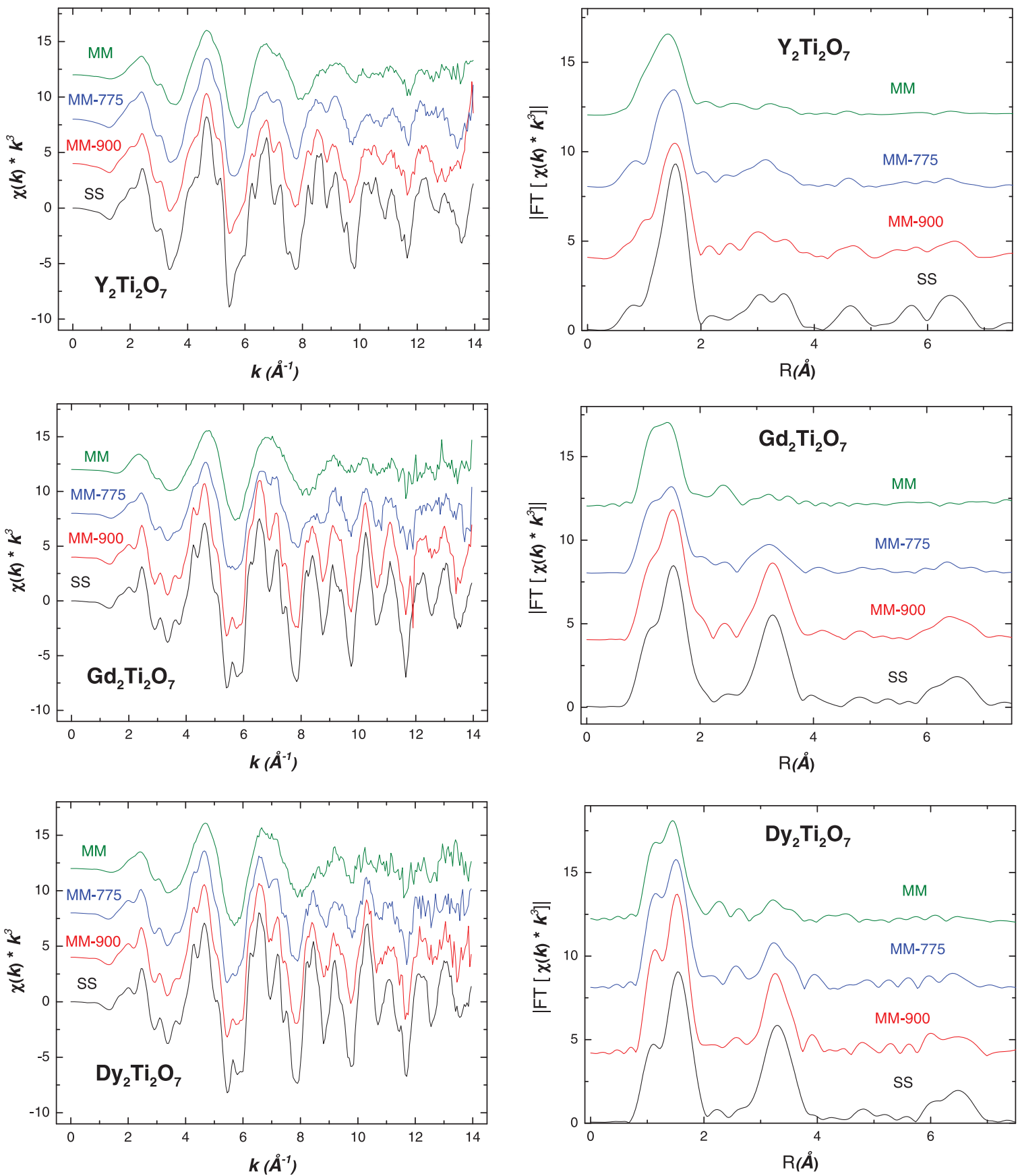

FIG. 9. (Color online) Comparison of the Ti $K$-edge $k^{3} \chi(k)$ EXAFS signals (left panel) and the modulus of their Fourier transforms in the case of MM and SS samples.

The computation performed by using the DH ECP potential and by imposing a $1 \%$ of overlapping factor yields the best reproduction of the whole absorption features. In particular, this calculation reproduces the three pre-peaks experimentally observed. Therefore, all the computations shown hereafter have been performed by using this ECP.

Once the XANES spectrum of SS $\mathrm{Gd}_{2} \mathrm{Ti}_{2} \mathrm{O}_{7}$ has been well reproduced by the computations we face the problem of determining the effect of disorder and/or the different Ti local environment in the MM samples. To this end we have performed the same class of computations as above but starting from the smallest $\mathrm{TiO}_{6}$ cluster, corresponding to photoabsorbing $\mathrm{Ti}$ and its first coordination shell, and by adding progressively further coordination shells. The results of these calculations, shown in Fig. 8, indicate that a good reproduction of the experimental spectrum requires a cluster of at least $5.45 \AA$ around Ti. For smaller clusters the reproduction of features $C_{1}$ to $C_{3}$ is not straightforward and, in addition, the pre-peak $A_{2}$ is not present in the theoretical simulation. The latter result indicates that the occurrence of a single pre-peak in the as-milled samples is not related to disorder effects affecting only the interatomic distances but maintaining fixed the $\mathrm{Ti}$ 
coordination. It rather suggests a lower Ti $\mathrm{CN}$. These results indicate that the samples obtained by mechanical milling show a great degree of structural disorder and, in addition, the $\mathrm{Ti}$ coordination decreases so that the nearest-neighbour Ti-O coordination polyhedron departs from the ideal pyrochlorelike one. The comparison of the spectra recorded on samples fired at $775^{\circ} \mathrm{C}$ and $900^{\circ} \mathrm{C}$ show great differences, indicating that the samples suffer a sudden disorder-order transition at a certain temperature between them. All these results thus indicate that the ordering process does not take place gradually and that the exothermic event observed in DTA is associated with an order-disorder transition.

These conclusions are corroborated by the comparison of the EXAFS spectra. Figure 9 shows the comparison of the Ti $K$-edge $\mathrm{k}^{3}$-weighted EXAFS and their Fourier transforms (FTs) for both MM and SS samples. In all the cases the EXAFS signal, $\chi(k)$, was obtained after removing the background by cubic spline polynomial fitting and by normalizing the magnitude of the oscillations to the edge jump. The corresponding pseudo-radial distribution function around the absorbing atom has been obtained by performing the Fourier transform (using a sine window) in the range $1.5 \leqslant \mathrm{k} \leqslant 13.5 \AA^{-1}$. Both EXAFS and FT signals show that in the case of the as-milled MM samples, despite the evidence of nanometric crystalline domains by XRD, the local ordering around $\mathrm{Ti}$ extends only to the first coordination shells. This local order slightly increases for the samples fired at $775^{\circ} \mathrm{C}$, and for the samples fired at the highest temperatures, it is similar to that of the samples obtained by traditional methods. It should be noted, however, that whereas in the case of DTO the 775-MM sample shows a local order approaching to that of the 900-MM and SS samples, the 775-MM GTO spectrum is similar to those of the as-milled $\mathrm{Y}$ and Dy samples, in agreement with both the XANES and Raman data. These results indicate that the long-range order sets in at different treatment temperatures for the three studied compounds.

Similar results are found in the analysis of Y $K$-edge, Gd $\mathrm{L}_{3}$-edge, and Dy $\mathrm{L}_{3}$-edge XANES and EXAFS spectra reported, respectively, in the supplementary material Figs. 15 and 16. These results indicate that in the case of the as-milled MM samples the disorder is similar at both A ( $\mathrm{Y}, \mathrm{Gd}$, and $\mathrm{Dy}$ ) and $\mathrm{B}$ (Ti) sites. In addition, the analysis of the $\mathrm{Dy}_{3}$-edge and Y $K$-edge signals also shows that in these cases the samples fired at $775{ }^{\circ} \mathrm{C}$ are significantly more ordered, approaching the ordered state shown by the 900-MM and SS samples, than in the case of GTO. In this respect it is important to note the evolution of the second peak of the Fourier transforms (at $\sim 3.3 \AA$ ) reported in the supplementary material, Fig. 16. This peak, due to the contribution of the atoms in the second coordination shell around the rare-earth and $\mathrm{Y}$ at the A sites, is already present in the as-milled YTO and DTO samples, but absent in the case of GTO. For both the Y and Dy cases its intensity strongly grows in the compounds fired at 775 ${ }^{\circ} \mathrm{C}$, while this enhancement occurs in the $\mathrm{Gd}$ case for firing temperatures of $900{ }^{\circ} \mathrm{C}$.

These conclusions are in agreement with the analysis of the XANES spectra recorded in situ as a function of the temperature (Fig. 10): the Ti $K$-edge XANES spectrum of the $\mathrm{Dy}_{2} \mathrm{Ti}_{2} \mathrm{O}_{7} \mathrm{MM}$ sample shows all the hallmarks of the SS compounds when heated at $\mathrm{T} \sim 660^{\circ} \mathrm{C}$. In contrast,
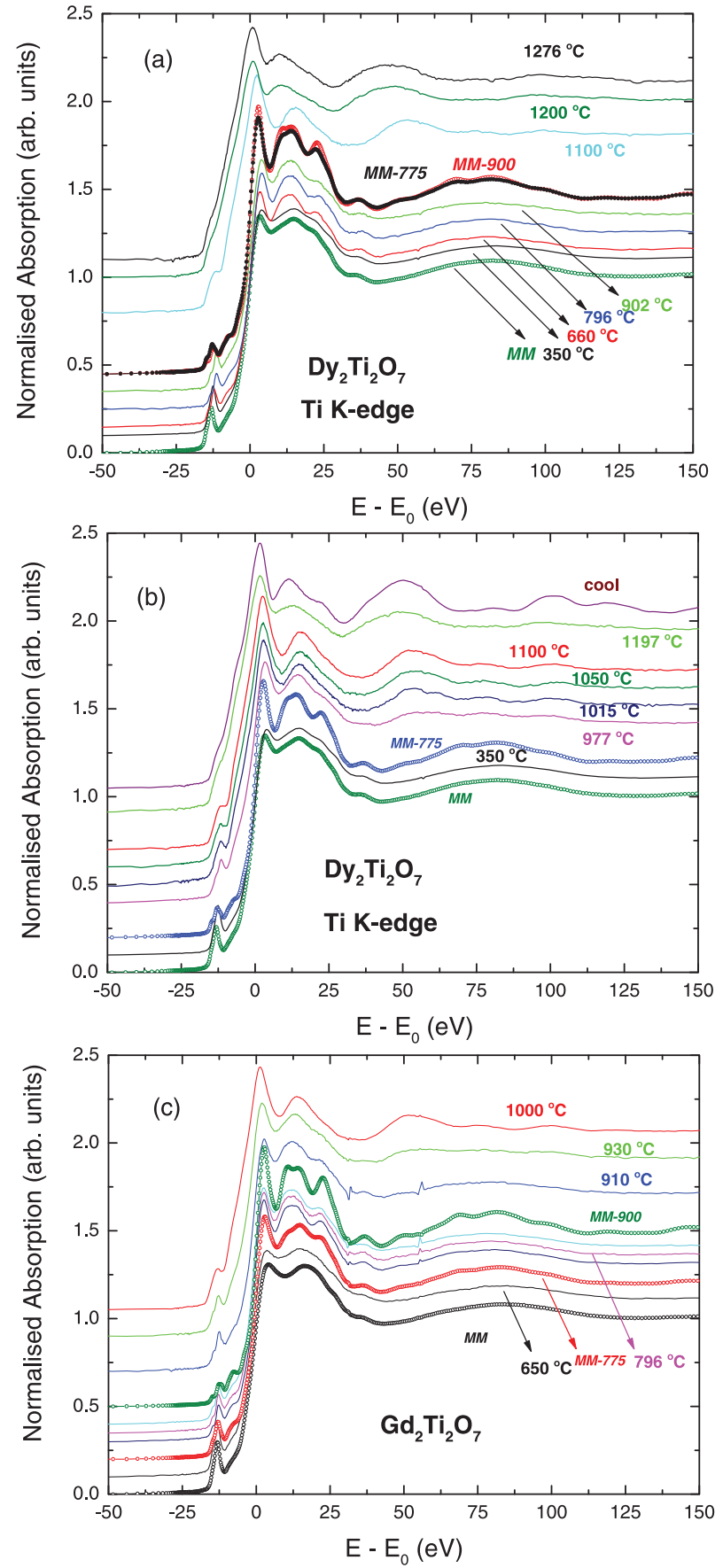

FIG. 10. (Color online) Comparison of the experimental Ti $K$ edge XANES spectrum recorded as a function of temperature in the case of the $\mathrm{MM} \mathrm{Dy}_{2} \mathrm{Ti}_{2} \mathrm{O}_{7}$ (a and b) $\mathrm{Gd}_{2} \mathrm{Ti}_{2} \mathrm{O}_{7}$ (c) samples.

the Gd compound does not show this ordering behavior up to temperatures close to $800^{\circ} \mathrm{C}$. For higher temperatures the spectra are similar in both cases, Gd and Dy, up to $\mathrm{T} \sim 1200^{\circ} \mathrm{C}$. For this temperature the absorption profile changes suggesting some reaction with the sample holder because after releasing temperature the XANES spectra do not correspond to any of those previously recorded for the studied samples. 

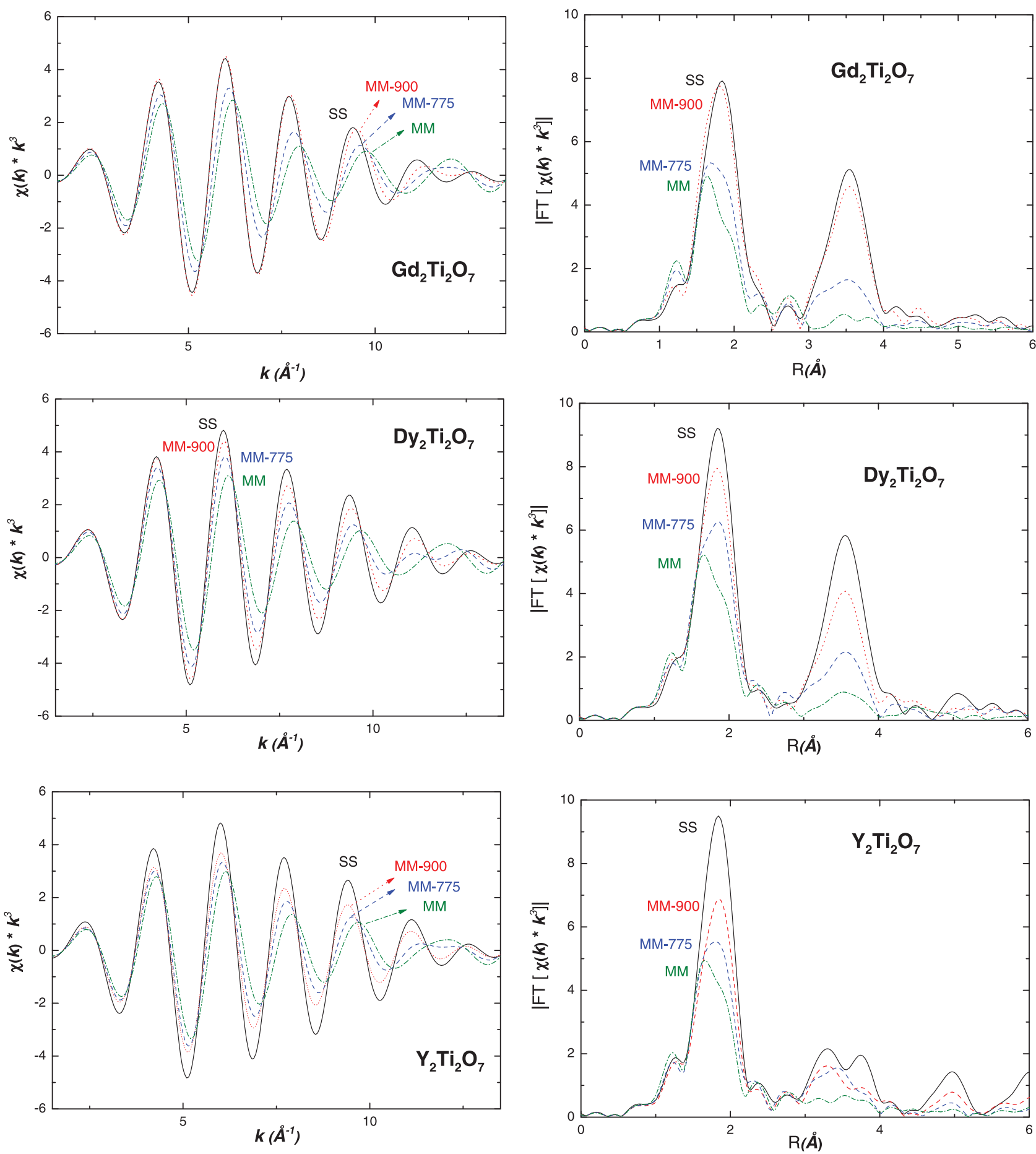

FIG. 11. (Color online) Dependence on the thermal treatment of the modulus of the Fourier transform and of the isolated contribution of the first coordination shell of the Ti $K$-edge $k^{3} \chi(k)$ EXAFS spectra of $\mathrm{Gd}_{2} \mathrm{Ti}_{2} \mathrm{O}_{7}, \mathrm{Y}_{2} \mathrm{Ti}_{2} \mathrm{O}_{7}$, and $\mathrm{Dy}_{2} \mathrm{Ti}_{2} \mathrm{O}_{7}$.

The picture emerging from the above characterization is that there is a significant local disorder at both $\mathrm{A}$ and $\mathrm{B}$ sites in the as cast samples. However, the disorder-order process progresses differently for the $\mathrm{Y}_{2} \mathrm{Ti}_{2} \mathrm{O}_{7}, \mathrm{Gd}_{2} \mathrm{Ti}_{2} \mathrm{O}_{7}$, and $\mathrm{Dy}_{2} \mathrm{Ti}_{2} \mathrm{O}_{7}$ samples. Trying to gain a deeper insight on the origin of this behavior we have analyzed in detail the variation of the first coordination shell of $\mathrm{Ti}$, common to all the compounds, as a function of the thermal treatment. We have isolated the EXAFS signal corresponding to this contribution by Fourier filtering and the comparison of the extracted signals, reported in Fig. 11, indicates that the average $\mathrm{Ti}-\mathrm{O}$ interatomic distance is shorter ( $\sim 0.05 \AA$ ) in the as-milled samples than in the SS ones (Table I, supplementary material). This interatomic distance becomes equal to that of the SS samples in the case of the fired YTO and DTO compounds. The same behavior is not observed in the GTO case until the sample is fired at $900{ }^{\circ} \mathrm{C}$. For this 
temperature the intensity of the first peak in the FT of GTO is more intense, and equal to that of the SS sample, than for $\mathrm{Y}$ and Dy compounds. Indeed, a closer inspection of the first peak of the FT suggests that for the MM samples the first Ti-O coordination shell contains two components whose relative weights vary with annealing temperature. These contributions may be attributed to two different environments, most likely with two different $\mathrm{CN}$. The long bond distance would arise from the usual quasioctahedral cage of B cations in pyrochlores, whereas the short distance may be attributed to a lower coordination, probably 5 or $5+1$. In the as-milled samples the short distance contribution prevails whereas the opposite is found for the 900-MM and SS samples. For 775-MM samples we find, again, a difference between GTO and the other two pyrochlores: for 775-MM DTO and YTO the intensity has already shifted to the long distance side, whereas for GTO the band corresponding to the short distance is still higher. These results point out that the as-milled MM $\mathrm{Gd}_{2} \mathrm{Ti}_{2} \mathrm{O}_{7}$ sample is the most disordered through the series. Moreover, the disorder-order transition takes place in this case at higher temperature than it does for $\mathrm{Y}_{2} \mathrm{Ti}_{2} \mathrm{O}_{7}$ and $\mathrm{Dy}_{2} \mathrm{Ti}_{2} \mathrm{O}_{7}$ compounds. In spite of this, its ordered phase is the most similar to that of the same pyrochlore obtained by solid state methods. These findings can be discussed in relation with the evolution of lattice parameters (inset, Fig. 2). Upon ordering, i.e., with increasing annealing temperature, lattice contracts in all three pyrochlores and so does the $\mathrm{R}-\mathrm{O}$ interatomic distance observed in EXAFS. In contrast, the average Ti-O distance expands. We attribute this expansion to the increase of the $\mathrm{Ti}$ coordination number. The difference between long-range and local structure behaviors supports our conclusion that each cation searches its ideal environment quite independently of the global lattice evolution.

The fact that the Ti-O interatomic distance is shorter in the MM samples than in the SS ones implies that the local environment of $\mathrm{Ti}$ atoms departs from that of the ideal pyrochlore structure. To asses this modification we have performed different $a b$ initio computations in which both the presence of vacancies and the occupancy of the $8 b$ site have been taken into account. The results of these computations are shown in Fig. 12. The agreement between the experimental and theoretical spectra worsens in all the models in which occupancy of the $8 b$ site is introduced, if no modification of the $48 f$ site is considered. In particular, the spectral features shift to lower energies and no variation of the pre-peaks region is observed, contrary to the experimental results. In contrast, the modification of the computed spectra follows the experimental trends when the coordination of $\mathrm{Ti}$ is changed from 6 to $5 \mathrm{O}$ atoms. It should be noted that the decrease in the coordination number should also imply a rearrangement of the Ti-O coordination polyhedra, i.e., interatomic distances and bond angles, which have not been considered in the calculations to avoid the arbitrary use of any free parameter.

Finally, we have also tested the presence of cation antisite defects in the as-milled samples. This disorder should imply that several rare-earth ions occupy the six-fold-coordinated B-site and, conversely, some $\mathrm{Ti}$ atoms occupy the 8-coordinated A sites. Therefore we have performed the theoretical computation of the Ti $K$-edge XANES spectra
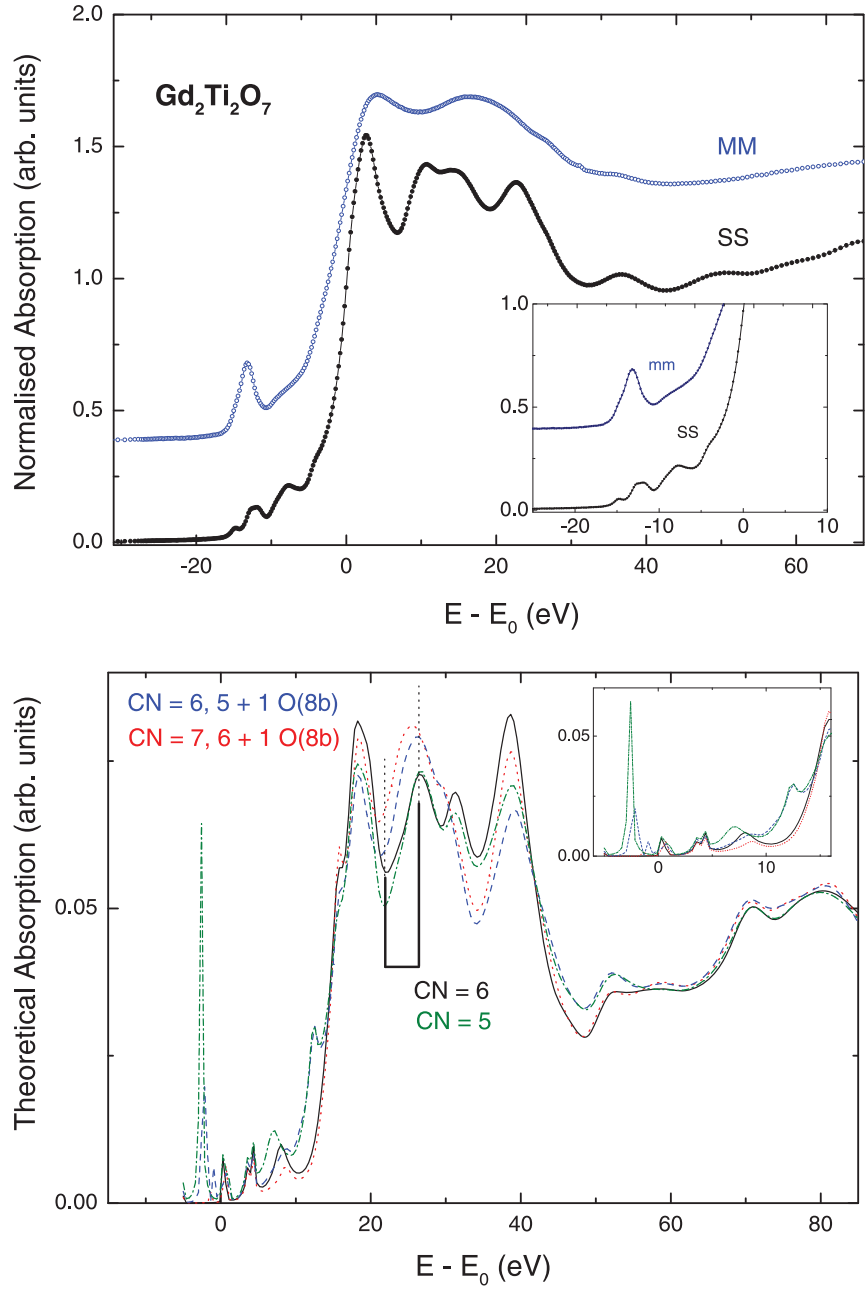

FIG. 12. (Color online) Comparison of the experimental Ti $K$ edge XANES spectra of the as-milled and SS samples of $\mathrm{Gd}_{2} \mathrm{Ti}_{2} \mathrm{O}_{7}$ (top panel) and the results of the calculations performed by considering different Ti-O structural arrangements (see text for details).

by considering that $\mathrm{Ti}$ occupies the $\mathrm{A}$ site. If this is the case, the experimental XANES spectra would correspond to the addition of contributions coming from photoabsorbing Ti atoms at both $\mathrm{A}$ and $\mathrm{B}$ sites. We have simulated this situation by adding the spectra computed for both classes of $\mathrm{Ti}$ atoms and weighting them according to the occupancy ratio of both crystallographic positions. The results of these computations, reported in Fig. 13, point out the existence of cation antisite defects in the as-milled samples. As the samples are subjected to the thermal treatment this chemical disorder decreases approaching to the ideal pyrochlore structure as for the SS samples. This finding is illustrated in Fig. 13 where computations performed by using different disorder degree are compared to the $\mathrm{Ti} K$-edge spectrum of the $\mathrm{Gd}_{2} \mathrm{Ti}_{2} \mathrm{O}_{7}$ sample fired at $775^{\circ} \mathrm{C}$. As discussed above, this sample displays a poor local order around $\mathrm{Ti}$ atoms that is similar to that of the as-milled $\mathrm{MM} \mathrm{Dy} \mathrm{Ti}_{2} \mathrm{O}_{7}$ and $\mathrm{Y}_{2} \mathrm{Ti}_{2} \mathrm{O}_{7}$ samples. The experimental spectrum is well accounted for by a computation in which $30 \%$ of the Ti atoms occupy the $\mathrm{Gd}$ sites. By decreasing the Ti occupancy of the A site the intensity 


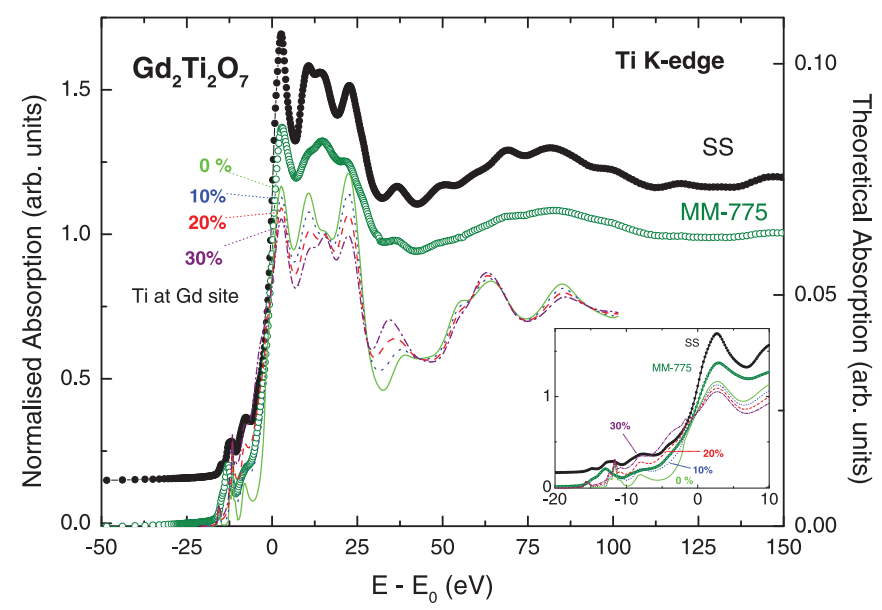

FIG. 13. (Color online) Comparison of the experimental Ti $K$ edge XANES spectra of the SS and MM sample fired $775^{\circ} \mathrm{C}$ $\mathrm{Gd}_{2} \mathrm{Ti}_{2} \mathrm{O}_{7}$ samples and the results of the calculations performed by considering $\mathrm{Gd} / \mathrm{Ti}$ site interchange.

ratio of the different absorption peaks is strongly modified and the overall spectral shape clearly evolves toward that of the ordered SS pyrochlores.

\section{SUMMARY AND CONCLUSIONS}

We have performed a combined Raman and x-ray absorption spectroscopy study of the $\mathrm{R}_{2} \mathrm{Ti}_{2} \mathrm{O}_{7}$ pyrochlores $(\mathrm{R}=\mathrm{Y}$, $\mathrm{Gd}$, and Dy) obtained by mechanical milling and by solid state reaction methods. With the aim of studying the nature of an exothermic event previously observed in DTA experiments, MM samples were submitted to annealing treatments at $775^{\circ} \mathrm{C}$ and $900^{\circ} \mathrm{C}$. In addition, both Raman and $\mathrm{x}$-ray absorption measurements have been recorded by performing an in situ thermal treatment in the range from ambient to $1300^{\circ} \mathrm{C}$ on the $\mathrm{MM}$ pyrochlore samples.

Raman results show that the as-milled $\mathrm{Y}_{2} \mathrm{Ti}_{2} \mathrm{O}_{7}$ and $\mathrm{Dy}_{2} \mathrm{Ti}_{2} \mathrm{O}_{7}$ samples are (highly defective) pyrochlores, while as-milled $\mathrm{Gd}_{2} \mathrm{Ti}_{2} \mathrm{O}_{7}$ shows a quasi amorphous configuration. The existence of a significant degree of structural disorder in the as-milled compounds is confirmed by both XANES and EXAFS spectra recorded at the Ti and Y $K$-edge and at the Gd and Dy $\mathrm{L}_{3}$-edges, which show that the local structure in the as-milled samples is highly distorted so that order extends only to the first coordination shells around the absorbing atoms. This is not in contradiction with the presence of diffraction peaks in XRD and pyrochlore-like bands in the Raman spectrum, and should be ascribed to the higher sensitivity of XANES and EXAFS to local atomic distribution. It should be noted in this respect that while XAS is a short-range technique, and thus emphasizes the similarity of the next-neighbouring coordination around the selected cation (Ti or R) in all three as-milled samples, the characteristic length scale of Raman probe is greater and several unit cells of the given phase are investigated. The combined analysis of the XRD, Raman and XAS data suggests that the as-milled samples might be described as formed by pyrochlore domains embedded in a fluorite-like substructure, but with a shorter correlation length in GTO as compared with DTO and YTO. Size effects are accompanied by the presence of a high defect concentration, in the form of cation exchange and oxygen Frenkel pairs, as confirmed by simulations of the XANES spectra. An interesting result that stems from the analysis of Raman and XANES spectra and their comparison with XRD results is that, even in the most disordered samples, each cation tends to keep its natural pyrochlore-like $\mathrm{CN}$. In the case of $\mathrm{Ti}^{4+}$, this implies that the occupancy of the $8 b$ vacant site is made at the expense of nearby $48 f$ oxygen site population, to avoid a seven-fold coordination for Ti. As a consequence, the $\mathrm{Ti} \mathrm{CN}$ at the $\mathrm{B}$ site is, on the average, lower than 6 , with a mean $\mathrm{Ti}-\mathrm{O}$ bond distance shorter than in ordered pyrochlores. This explains the presence of a high-frequency band in the Raman spectra of samples below the DTA event, as well as the appearance of a single pre-peak in Ti-XANES spectra.

The evolution of Raman and XAS spectra upon thermal treatment shows that, although the three compounds evolve toward a long-range ordered lattice on approaching the DTA event, this process occurs at different rates for the three studies compounds, being faster for GTO once the pyrochlore lattice is already crystallized. Thus, although the as-milled $\mathrm{Gd}_{2} \mathrm{Ti}_{2} \mathrm{O}_{7}$ sample is almost amorphous and presents the disorder-order transition at higher temperature than both $\mathrm{Y}_{2} \mathrm{Ti}_{2} \mathrm{O}_{7}$ and $\mathrm{Dy}_{2} \mathrm{Ti}_{2} \mathrm{O}_{7}$, its ordered phase is the most similar to that of the same pyrochlore obtained by solid state methods, in agreement with the fact that this compound tolerates disorder in the pyrochlore phase to a much lesser extent than YTO or DTO.

The ordering event can be described as a thermally activated process involving cation and anion diffusion. According to in situ experiments, this process occurs in a narrow temperature range: between 750 and $800{ }^{\circ} \mathrm{C}$ in DTO and YTO, and between 850 and $900^{\circ} \mathrm{C}$ in GTO. The softening of the high-frequency Raman band at the ordering event indicates an increase of the mean Ti-O bond distance, in agreement with the EXAFS data, despite the global lattice contraction. Moreover, the pre-edge region of the Ti $K$-edge XANES changes from a single peak to the three-peaks structure characteristic of a six-coordinated $\mathrm{Ti}$ atom whose local coordination is well ordered beyond the second coordination shell. All these features are indicative of an increase of the $\mathrm{CN}$ around $\mathrm{Ti}$ from 5 (or $5+1$ ) to the value $\mathrm{CN}=6$ of ordered pyrochlores. Theoretical XANES computations demonstrate the existence of both antisite and Frenkel defects whose concentration decreases upon thermal treatment.

In conclusion, the as-milled $\mathrm{R}_{2} \mathrm{Ti}_{2} \mathrm{O}_{7}$ materials are already pyrochlore-like but afford a high defect concentration, and thus present small well ordered areas. Upon thermal treatment the defect concentration decreases rapidly on approaching the ordering event, resulting in an enlargement of pyrochlore-like domains through a nucleation and growth phenomenon. This process is thermally activated and the "event" observed in DTA can be seen as a transition from short/medium-range (tens of $\mathrm{nm}$ ) to long-range (hundreds of $\mathrm{nm}$ ) ordering of both cations and anions. 


\section{ACKNOWLEDGMENTS}

This work was partially supported by Spanish CICYTMAT2008-06542-C04 and MAT2010-19837-C06-06 grants. The synchrotron radiation experiments were performed at Elettra (Proposal No. 20095026). C. Guglieri acknowledges the Ministerio de Eduación y Ciencia of Spain for a PhD. Grant. The authors would like to thank Daniel T. Bowron for critical reading of the paper.

\section{SUPPLEMENTARY MATERIAL}

Through the computations we have tested the performance of different exchange and correlation potentials (ECP) to account for the experimental spectrum. In this way we have used both energy-dependent Hedin-Lundqvist (HL) and DiracHara ECP potentials. ${ }^{13,14}$ The HL is a complex potential in which its imaginary part accounts for the inelastic losses of the photoelectron. However, it has been reported that in several cases the damping of the signal simulated in this way is excessive. ${ }^{15,42,43}$ Therefore, we have also made computations by using only the real part of the HL ECP (hereafter, real HL). Conversely, because DH is a real ECP we have built a "complex" DH one by adding to the standard energydependent Dirac-Hara exchange potential the imaginary part of the HL ECP. The results, reported in Fig. 14 shows a good agreement with the experimental data. As previously noted, the imaginary part of the HL ECP introduces an excessive and unrealistic damping of the signal resulting in the smoothing of the main absorption line (peak $\mathrm{B}$ ) and of the peaks forming the first broad resonance $\left(\mathrm{C}_{1}, \mathrm{C}_{2}, \mathrm{C}_{3}\right)$. However, the relative intensity of the high energy spectral features ( $D$ to $F$ ) is nicely reproduced by these computations. This result is due to the fact that for these higher energies the imaginary part of the ECP actuates as an effective Debye-Waller factor attenuating the EXAFS amplitude. By contrast, computations performed by using real ECP yield a good reproduction of the whole absorption feature.

The Ti $K$-edge EXAFS signals were obtained according to standard procedures. ${ }^{10}$ The pseudoradial distribution function around $\mathrm{Ti}$ atoms has been obtained by $\mathrm{k}^{3}$-weighting the EXAFS in the range $2.7 \leqslant \mathrm{k} \leqslant 13.2 \AA^{-1}$, multiplied by a Hanning window and Fourier transforming (FT). The contribution of the first coordination Ti-O shell was obtained by Fourier filtering the signals in the range $0.8 \leqslant R \leqslant 2.5 \AA$. For a comparative study, the Fourier transform of the
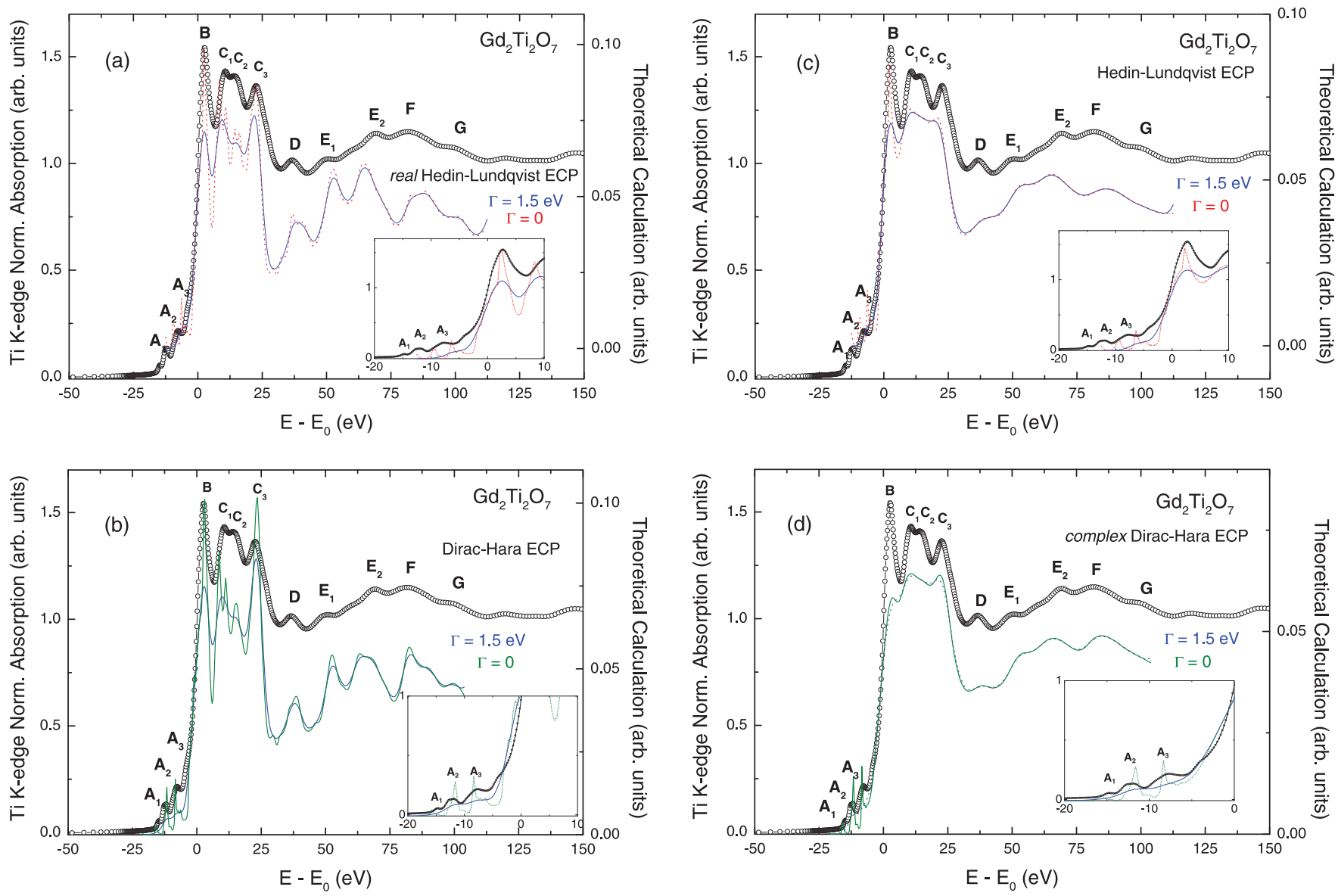

FIG. 14. (Color online) Comparison of the experimental Ti $K$-edge XANES spectrum of $\mathrm{Gd}_{2} \mathrm{Ti}_{2} \mathrm{O}_{7}(\circ)$ and the results of the calculations performed by using different ECP potentials: (a) real HL, (b) DH, (c) HL, and (d) complex DH. In all the cases the dotted lines correspond to the convolution of the theoretical spectra with a Lorentzian shape function to account for the core-hole lifetime. 


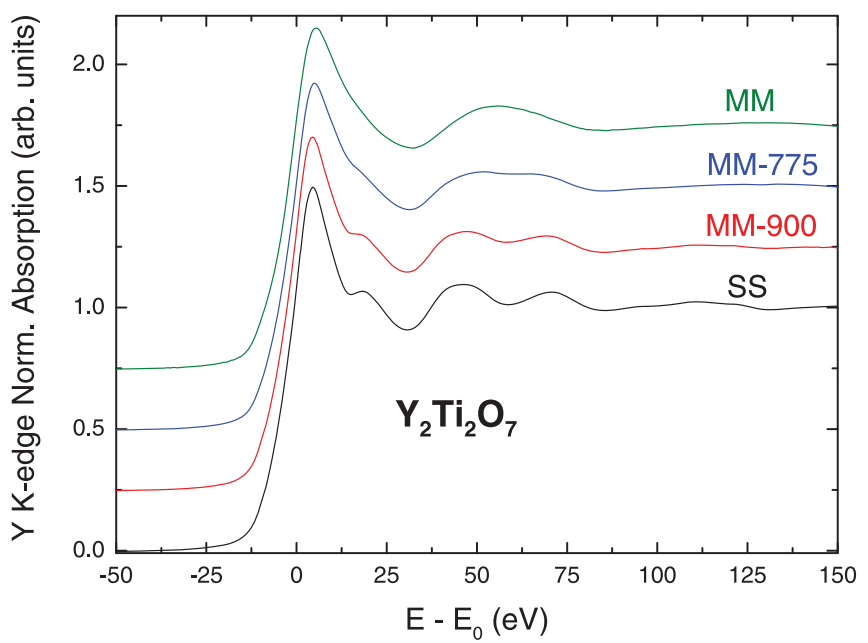

TABLE I. Best fit parameters obtained from the analysis of the first shell contribution to the Ti $K$-edge EXAFS spectrum in $\mathrm{R}_{2} \mathrm{Ti}_{2} \mathrm{O}_{7}$ pyrochlores $(\mathrm{R}=\mathrm{Gd}$, Dy, and $\mathrm{Y})$.

\begin{tabular}{|c|c|c|c|c|}
\hline Model & $\begin{array}{l}\mathrm{N}_{\text {Coord }} \\
\text { fixed }\end{array}$ & $\begin{array}{c}\mathrm{r}_{\mathrm{Ti}-\mathrm{O}} \\
\AA\end{array}$ & $\begin{array}{c}\Delta \sigma_{\mathrm{Ti}-\mathrm{O}}^{2} \\
\AA^{2}\end{array}$ & $\mathcal{R}$ \\
\hline $\mathrm{Gd}_{2} \mathrm{Ti}_{2} \mathrm{O}_{7}$ & 6 & 1.95 & & \\
\hline MM-900 & 6 & $1.94 \pm 0.01$ & $0.000 \pm 0.001$ & 0.06 \\
\hline MM-775 & 6 & $1.92 \pm 0.01$ & $0.004 \pm 0.001$ & 0.09 \\
\hline MM-775 & 5 & $1.91 \pm 0.01$ & $0.002 \pm 0.001$ & 0.07 \\
\hline MM & 6 & $1.89 \pm 0.02$ & $0.007 \pm 0.002$ & 0.16 \\
\hline MM & 5 & $1.88 \pm 0.02$ & $0.005 \pm 0.002$ & 0.13 \\
\hline $\mathrm{Dy}_{2} \mathrm{Ti}_{2} \mathrm{O}_{7}$ & 6 & 1.94 & & \\
\hline MM-900 & 6 & $1.94 \pm 0.01$ & $0.002 \pm 0.001$ & 0.03 \\
\hline MM-900 & 5 & $1.93 \pm 0.01$ & $0.001 \pm 0.001$ & 0.03 \\
\hline MM-775 & 6 & $1.92 \pm 0.01$ & $0.004 \pm 0.001$ & 0.04 \\
\hline MM-775 & 5 & $1.92 \pm 0.01$ & $0.003 \pm 0.001$ & 0.04 \\
\hline MM & 6 & $1.90 \pm 0.02$ & $0.007 \pm 0.002$ & 0.13 \\
\hline MM & 5 & $1.88 \pm 0.02$ & $0.005 \pm 0.002$ & 0.09 \\
\hline $\mathrm{Y}_{2} \mathrm{Ti}_{2} \mathrm{O}_{7}$ & 6 & 1.96 & & \\
\hline MM-900 & 6 & $1.96 \pm 0.01$ & $0.003 \pm 0.001$ & 0.05 \\
\hline MM-900 & 5 & $1.96 \pm 0.01$ & $0.001 \pm 0.001$ & 0.08 \\
\hline MM-775 & 6 & $1.94 \pm 0.01$ & $0.005 \pm 0.001$ & 0.09 \\
\hline MM-775 & 5 & $1.93 \pm 0.01$ & $0.003 \pm 0.001$ & 0.06 \\
\hline MM & 6 & $1.91 \pm 0.02$ & $0.006 \pm 0.002$ & 0.16 \\
\hline MM & 5 & $1.91 \pm 0.02$ & $0.005 \pm 0.002$ & 0.13 \\
\hline
\end{tabular}

${ }^{\mathrm{a}} \Delta \mathrm{k}=2.7-13.2 \AA^{-1} ; \Delta \mathrm{R}=0.8-2.5 \AA ; \mathrm{N}_{\text {Coord }}$, coordination number; $\mathrm{d}$, interatomic distance; $\sigma^{2}$, Debye-Waller factor; $\mathcal{R}$, reliability factor. ${ }^{44}$

EXAFS signals was performed within the same $\mathrm{k}$ range. The EXAFS signals of the samples obtained by mechanical milling were analyzed using the phase and amplitude transferability method. The EXAFS simulation was performed using the VIPER program. ${ }^{44}$ The backscattering amplitude and phase were obtained from the EXAFS spectra of the SS samples. In this way the only parameters allowed to vary during the fitting procedure were the Ti-O interatomic distance, $\mathrm{r}_{\mathrm{Ti}-\mathrm{O}}$, and the Debye-Waller factor, $\sigma^{2}$, relative to that of the corresponding SS sample. The fittings were made by imposed integer coordination number, $\mathrm{N}_{\text {Coord }}$, values. 

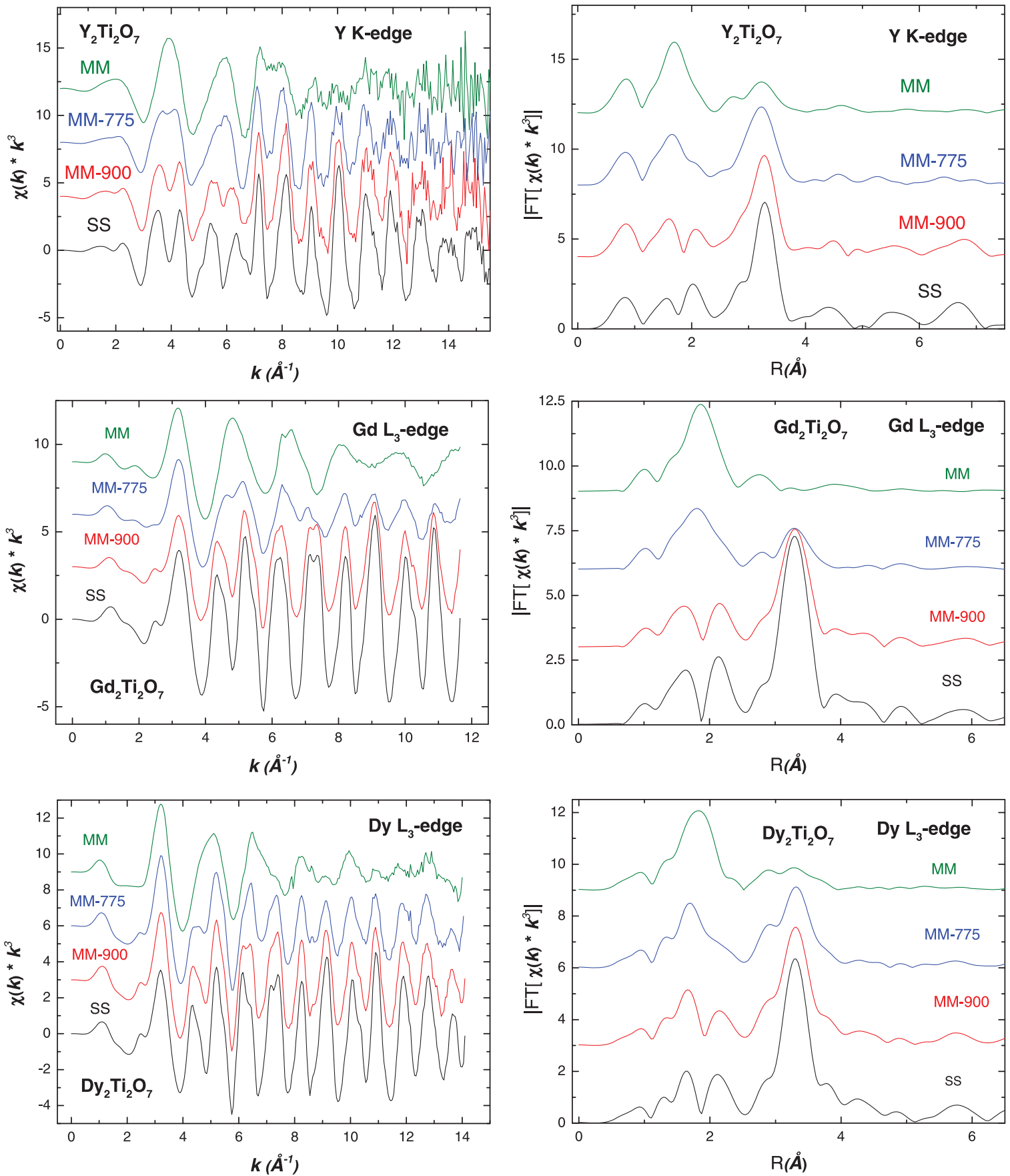

FIG. 16. (Color online) Comparison of the $\mathrm{Y} K$-edge, $\mathrm{Gd} \mathrm{L}_{3}$-edge, and Dy $\mathrm{L}_{3}$-edge $k^{3} \chi(k)$ EXAFS signals (left panel) and the modulus of their Fourier transforms in the case of MM and SS samples: $\mathrm{Y}_{2} \mathrm{Ti}_{2} \mathrm{O}_{7}$ (top panel), $\mathrm{Gd}_{2} \mathrm{Ti}_{2} \mathrm{O}_{7}$ (middle), and $\mathrm{Dy}_{2} \mathrm{Ti}_{2} \mathrm{O}_{7}$ (bottom).

*jchaboy@unizar.es

${ }^{1}$ B. J. Wuensch, K. W. Eberman, C. Heremans, E. M. Ku, P. Onnerud, E. M. E. Yeo, S. M. Haile, J. K. Stalick, and J. D. Jorgensen, Solid State Ionics 129, 111 (2000).

${ }^{2}$ J. Snyder, J. S. Slusky, R. J. Cava, and P. Schiffer, Nature 413, 48 (2001).

${ }^{3}$ J. Wu, X. Wen, N. P. Padture, P. G. Klemens, M. Gell, E. Garcia, P. Miranzo, and M. I. Osendi, J. Am. Ceram. Soc. 85, 3031 (2002).
${ }^{4}$ K. E. Sickafus, L. Minervini, R. W. Grimes, J. A. Valdez, M. Ishimaru, F. Li, K. J. McClellan, and T. Hartmann, Science 289, 748 (2000).

${ }^{5}$ C. Suryanarayana, Prog. Mater. Sci. 46, 1 (2001).

${ }^{6}$ G. García-Martínez, L. G. Martínez-González, J. I. EscalanteGarcía, and A. F. Fuentes, Powder Technol. 152, 72 (2005).

${ }^{7}$ A. F. Fuentes, K. Boulahya, M. Maczka, J. Hanuza, and U. Amador, Solid State Sci. 7, 343 (2005). 
${ }^{8}$ A. D. Cicco, G. Aquilanti, M. Minicucci, E. Principi, N. Novello, A. Cognigni, and L. Olivi, J. Phys.: Conf. Ser. 190, 012043 (2009).

${ }^{9}$ D. E. Sayers and B. A. Bunker, EXAFS data analysis, Chapter 6 in Extended X-Ray Absorption Fine Structure, edited by R. Prins and D. Koningsberger (Wiley, New York, 1988).

${ }^{10}$ B. Ravel and M. Newville, J. Synchrotron Radiat. 12, 537 (2005).

${ }^{11}$ C. R. Natoli and M. Benfatto (unpublished).

${ }^{12}$ C. R. Natoli and M. Benfatto, J. Phys. (Paris) Colloq. 47, C8 (1986).

${ }^{13}$ J. Chaboy and S. Quartieri, Phys. Rev. B 52, 6349 (1995).

${ }^{14}$ J. Chaboy, J. Synchrotron Radiat. 16, 533 (2009).

${ }^{15}$ J. Chaboy, N. Nakajima, and Y. Tezuka, J. Phys. Condens. Matter 19, 266206 (2007).

${ }^{16}$ M. O. Krause and J. H. Oliver, J. Phys. Chem. Ref. Data 8, 329 (1979).

${ }^{17}$ M. A. Subramanian, G. Aravamudan, and G. V. Subba Rao, Prog. Solid State Chem. 15, 55 (1983).

${ }^{18}$ M. Maczka, J. Hanuza, K. Hermanowicz, A. F. Fuentes, K. Matsuhira, and Z. Hiroi, J. Raman Spectrosc. 39, 537 (2008).

${ }^{19}$ M. Maczka, M. L. Sanjuán, A. F. Fuentes, L. Macalik, J. Hanuza, K. Matsuhira, and Z. Hiroi, Phys. Rev. B 79, 214437 (2009).

${ }^{20}$ H. C. Gupta, S. Brown, N. Rani, and V. B. Gohel, J. Raman Spectrosc. 32, 41 (2001).

${ }^{21}$ H. C. Gupta, J. Singh, S. Kumar, Karandeep, and N. Rani, J. Mol. Struct. 937, 136 (2009).

${ }^{22}$ F. Zhang, J. Wang, M. Lang, J. Zhang, and R. Ewing, J. Solid State Chem. 183, 2636 (2010).

${ }^{23}$ M. Lang, F. X. Zhang, R. C. Ewing, J. Lian, C. Trautmann, and Z. Wang, J. Mater. Res. 24, 1322 (2009).

${ }^{24}$ M. Lang, F. Zhang, J. Zhang, J. Wang, J. Lian, W. J. Weber, B. Schuster, C. Trautmann, R. Neumann, and R. C. Ewing, Nucl. Instrum. Methods Phys. Res., Sect. B 268, 2951 (2010).

${ }^{25}$ M. Glerup, O. F. Nielsen, and F. W. Poulsen, J. Solid State Chem. 160, 25 (2001).

${ }^{26}$ N. J. Hess, B. D. Begg, S. D. Conradson, D. E. McCready, P. L. Gassman, and W. J. Weber, J. Phys. Chem. B 106, 4663 (2002).

${ }^{27}$ F. X. Zhang and S. K. Saxena, Chem. Phys. Lett. 413, 248 (2005).

${ }^{28}$ C. Heremans, B. J. Wuensch, J. K. Stalick, and E. Prince, J. Sol. State Chem. 117, 108 (1995).

${ }^{29}$ G. Burns, F. H. Dacol, G. Kliche, W. Konig, and M. W. Shafer, Phys. Rev. B 37, 3381 (1988).

${ }^{30}$ M. A. Laguna, M. L. Sanjuán, A. Várez, and J. Sanz, Phys. Rev. B 66, 054301 (2002).

${ }^{31}$ M. T. Paques-Ledent, Spectrochim. Acta, Part A: Molecular Spectroscopy 32, 1339 (1976).
${ }^{32}$ J. Xu, A. P. Wilkinson, and S. Pattanaik, Chem. Mater. 12, 3321 (2000).

${ }^{33}$ In addition to the band centered at $750 \mathrm{~cm}^{-1}$, another disorder activated band appears at $600 \mathrm{~cm}^{-1}$, which can be differentiated from the $\mathrm{T}_{2 g}$ allowed mode at $\sim 580 \mathrm{~cm}^{-1}$ by its frequency and intensity. Using the same type of arguments used to interpret the hf band, the band at $600 \mathrm{~cm}^{-1}$ has to be attributed to oxygen in Ti environments with higher $\mathrm{CN}$, probably $\mathrm{CN}=6$. We remind that the breathing mode of $\mathrm{TiO}_{6}$ octahedra is not an allowed mode in pyrochlores, so that as the lattice becomes more pyrochlore-like the mode becomes progressively forbidden.

${ }^{34}$ J. Lian, L. Wang, J. Chen, K. Sun, R. C. Ewing, J. M. Farmer, and L. A. Boatner, Acta Mater. 51, 1493 (2003).

${ }^{35}$ L. Minervini and R. W. Grimes, J. Am. Ceram. Soc. 83, 1873 (2000).

${ }^{36}$ W. R. Panero, L. Stixrude, and R. C. Ewing, Phys. Rev. B 70, 054110 (2004).

${ }^{37}$ Heremans et al. conclude in [28] that anion disorder in $\mathrm{Y}_{2}(\mathrm{Ti}$, $\mathrm{Zr})_{2} \mathrm{O}_{7}$ is independent of cation disorder and, moreover, $\mathrm{O}_{1}$ diffusion preceding that of $\mathrm{O}_{2}$. However, these results deserve some comments: according to their neutron diffraction results the occupation of the $\mathrm{O}_{3}$ site starts, at the expense of $\mathrm{O}_{1}$ ions, before $\mathrm{A} / \mathrm{B}$ cation exchange occurs. It has to be noted that $\mathrm{Ti} / \mathrm{Zr}$ mixed pyrochlores have intrinsic cation disorder at the $\mathrm{B}$ sites and then, the considerations about $\mathrm{CN}$ preference of large or small cations apply. Consequently, the $\mathrm{O}_{1}$ vacancies and $\mathrm{O}_{3}$ occupation are correlated to cation disorder at the B site. Similarly, the onset of disorder at the $\mathrm{O}_{2}$ sublattice is also correlated to cation disorder, this time in the form of $\mathrm{A} / \mathrm{B}$ exchange. Thus, disorder at the anion and cation sublattices are not independent, though they may affect differently the $\mathrm{O}_{1}$ and $\mathrm{O}_{2}$ sublattices depending on the kinetics and size of the constituting cations.

${ }^{38}$ T. Moriga, S. Emura, A. Yoshiasa, S. Kikkawa, F. Kanamaru, and K. Koto, Solid State Ionics 40-41, 357 (1990).

${ }^{39}$ D. Michel, M. Pérez y Jorba, and R. Collongues, J. Raman Spectrosc. 5, 163 (1976).

${ }^{40}$ T. Uehara, K. Koto, S. Emura, and F. Kanamaru, Solid State Ionics 23, 331 (1987).

${ }^{41}$ F. Farges, G. E. Brown, and J. J. Rehr, Phys. Rev. B 56, 1809 (1997).

${ }^{42}$ J. Chaboy, H. Maruyama, and N. Kawamura, J. Phys. Condens. Matter 19, 216214 (2007).

${ }^{43}$ K. Hatada and J. Chaboy, Phys. Rev. B 76, 104411 (2007).

${ }^{44}$ K. V. Klementiev, J. Phys. D: Appl. Phys. 34, 209 (2001). 